\title{
Gauge-Yukawa theories: Beta functions at large $N_{f}$
}

\author{
Oleg Antipin, ${ }^{1, *}$ Nicola Andrea Dondi, ${ }^{2, \dagger}$ Francesco Sannino, ${ }^{2, *}$ Anders Eller Thomsen, ${ }^{2, \S}$ and Zhi-Wei Wang ${ }^{2,3, \|}$ \\ ${ }^{1}$ Rudjer Boskovic Institute, Division of Theoretical Physics, Bijenička 54, HR-10000 Zagreb, Croatia \\ ${ }^{2} C P^{3}$-Origins, University of Southern Denmark, Campusvej 55, 5230 Odense M, Denmark \\ ${ }^{3}$ Department of Physics, University of Waterloo, Waterloo, Ontario N2L 3G1, Canada
}

(Received 17 April 2018; published 6 July 2018)

\begin{abstract}
We consider the dynamics of gauge-Yukawa theories in the presence of a large number of matter constituents. We first review the current status for the renormalization group equations of gauge-fermion theories and extend the results to semisimple groups. In this regime these theories develop an interacting ultraviolet fixed point that for the semisimple case leads to a rich phase diagram. The latter contains a complete asymptotically safe fixed point repulsive in all couplings. We then add two gauged Weyl fermions belonging to arbitrary representations of the semisimple gauge group and a complex, gauged scalar to the original gauge-fermion theory allowing for new Yukawa interactions and quartic scalar self-coupling. Consequently, we determine the first nontrivial order in $1 / N_{f}$ for the Yukawa and quartic beta functions. Our work elucidates, consolidates, and extends results obtained earlier in the literature. We also acquire relevant knowledge about the dynamics of gauge-Yukawa theories beyond perturbation theory. Our findings are applicable to any extension of the standard model featuring a large number of fermions such as asymptotic safety.
\end{abstract}

DOI: 10.1103/PhysRevD.98.016003

\section{INTRODUCTION}

The most general classes of four-dimensional, renormalizable quantum field theories (QFTs) are in the form of gauge-Yukawa theories. Their dynamics underlies the standard model (SM) interactions and those of any of its sensible extensions. It is therefore paramount to gain a deeper understanding of their dynamics, which is often limited to perturbation theory.

Fundamental theories are those gauge-Yukawa theories that, according to Wilson [1,2], are well defined at arbitrarily short distances. Asymptotically free [3,4] and safe [5] QFTs are complementary examples of fundamental theories. ${ }^{1}$ The recent discovery of four-dimensional, controllable, in the perturbative sense, asymptotically safe QFTs [5,7] has opened the way to novel dark and bright extensions of the SM [8-15]. More generally, it is interesting to investigate

*antipin@irb.hr
oandondi@cp3.sdu.dk
*sannino@cp3.sdu.dk
\$aethomsen@cp3.sdu.dk
$\|_{\text {wang@cp3.sdu.dk }}$
${ }^{1}$ The instanton analysis and contribution can be found in [6].

Published by the American Physical Society under the terms of the Creative Commons Attribution 4.0 International license. Further distribution of this work must maintain attribution to the author(s) and the published article's title, journal citation, and DOI. Funded by SCOAP ${ }^{3}$. the short distance fate of the SM and its extensions including gravity [16-20].

To gain information beyond perturbation theory, one can use supersymmetry. A systematic investigation of nonperturbative constraints that a supersymmetric, asymptotically safe QFT must abide, including a-maximization [21] and collider bounds [22], appeared in [23] extending and correcting the results of [24]. Building upon results of [21], the first evidence for nonperturbative, supersymmetric safety was gathered in [25] and further analyzed in [26].

Nonperturbative results can also be deduced for nonsupersymmetric theories when considering specific limits in theory space: for example, building upon the large $N_{f}$ results of [27-31], that gauge-fermion theories at any finite number of colors can be argued to develop a nonperturbative ultraviolet (UV) fixed point [32]. Consequently, one can extend the original conformal window, reviewed in $[33,34]$, to include an asymptotically safe phase [32].

It is therefore timely to consider the dynamics of gaugeYukawa theories at large $N_{f}$ [14,35-37]. We investigate this here by elucidating, consolidating, and extending the results obtained earlier in the literature. The results are useful when searching for asymptotically safe extensions of the SM $[13,14]$.

The paper is organized as follows. In Sec. II, we introduce our model and the renormalization conventions used throughout the paper. Section III then proceeds to review the current status of large $N_{f}$ computations for the gauge beta function. We then generalize the results to 
semisimple gauge groups and in addition we present their phase diagrams. In Sec. IV, we provide a detailed computation of the Yukawa and quartic coupling beta functions at the first nontrivial order in $1 / N_{f}$ for generic, semisimple gauge groups. Section V concludes the paper. The explicit derivations of the various resummation formulas used in the large $N_{f}$ computations can be found in Appendix.

\section{GAUGE-YUKAWA MODELS: NOTATION AND CONVENTIONS}

We consider both Abelian and non-Abelian semisimple gauge-Yukawa models featuring $N_{f}$ vectorlike fermions, $\Psi_{I}$ charged under the full gauge group. Additionally, the models contain two Weyl spinors, $\chi$ and $\xi$, and a complex scalar, $\phi$, such that there is enough content to form a (chiral) Yukawa coupling among these three fields, and for quartic scalar selfinteractions to emerge. ${ }^{2}$ The field content of the model is summarized in Table I where we report the transformation of each matter field with respect to the gauge interactions.

The Lagrangian of the theory reads

$$
\begin{aligned}
& \mathcal{L}=-\frac{1}{4} F_{\mu \nu}^{A} F^{A, \mu \nu}+\sum_{I=1}^{N_{f}} i \bar{\Psi}_{I} \gamma^{\mu} D_{\mu} \Psi^{I}+i \bar{\chi} \bar{\sigma}^{\mu} D_{\mu} \chi \\
& +i \bar{\xi} \bar{\sigma}^{\mu} D_{\mu} \xi+\left(D_{\mu} \phi\right)^{\dagger}\left(D_{\mu} \phi\right) \\
& -\left(y_{a i j} \phi^{a} \chi^{i} \xi^{j}+y^{*, a i j} \phi_{a}^{*} \bar{\chi}_{i} \bar{\xi}_{j}\right)-\frac{1}{4} \lambda^{a b}{ }_{c d} \phi_{a}^{*} \phi_{b}^{*} \phi^{c} \phi^{d},
\end{aligned}
$$

where the index $I=1, \ldots N_{f}$ is the $\Psi$ flavor index, $i, j$ are gauge indices for $\chi$ and $\xi$, and $a, b, c, d$ are reserved for the gauged scalar indices within a given representation that can be read off from the associated covariant derivative

$$
\begin{aligned}
D_{\mu} \Psi_{I}^{i} & =\left[\partial_{\mu}+i g A_{\mu}^{A}\left(T_{\Psi}^{A}\right)^{i}{ }_{j}\right] \Psi_{I}^{j}, \\
D_{\mu} \chi^{i} & =\left[\partial_{\mu}+i g A_{\mu}^{A}\left(T_{\chi}^{A}\right)^{i}{ }_{j}\right] \chi^{j}, \\
D_{\mu} \xi^{i} & =\left[\partial_{\mu}+i g A_{\mu}^{A}\left(T_{\xi}^{A}\right)^{i}{ }_{j}\right] \xi^{j}, \\
D_{\mu} \phi^{a} & =\left[\partial_{\mu}+i g A_{\mu}^{A}\left(T_{\phi}^{A}\right)_{b}^{a}\right] \phi^{b} .
\end{aligned}
$$

In the most general version of the model, the gauge group is allowed to be semisimple. The generalization of the covariant derivative in this case is straightforward. Gauge invariance imposes the following constraints,

$$
\begin{aligned}
0= & y_{b i j}\left(T_{\phi}^{A}\right)^{b}{ }_{a}+y_{a k j}\left(T_{\chi}^{A}\right)^{k}{ }_{i}+y_{a i k}\left(T_{\xi}^{A}\right)^{k}{ }_{j}, \\
0= & -\lambda^{e b}{ }_{c d}\left(T_{\phi}^{* A}\right)_{e}{ }^{a}-\lambda^{a e}{ }_{c d}\left(T_{\phi}^{* A}\right)_{e}{ }^{b}+\lambda^{a b}{ }_{e d}\left(T_{\phi}^{A}\right)^{e}{ }_{c} \\
& +\lambda^{a b}{ }_{c e}\left(T_{\phi}^{A}\right)^{e}{ }_{d},
\end{aligned}
$$

\footnotetext{
${ }^{2}$ Gauge anomalies are avoided by either adding new chiral fermions or by arranging $\chi, \xi$ in anomaly-free representations of the gauge group. Our results are adaptable to a given gaugeanomaly-free model.
}

TABLE I. Summary of the field content of the model. The first two columns detail the transformation of each field under Lorentz and flavor symmetry. $q_{\Psi, \chi, \xi, \phi}$ denotes the U(1) charges of the fields, while $R_{\Psi, \chi, \xi, \phi}^{\alpha}$ are the representation of the fields under each simple gauge group labeled by $\alpha$.

\begin{tabular}{lcccc}
\hline \hline Fields & $\mathrm{SO}(1,3)^{+}$ & $\mathrm{SU}\left(N_{f}\right)$ & $\mathrm{U}(1)$ & $\times_{\alpha} G_{\alpha}$ \\
\hline$\Psi$ & $\left(\frac{1}{2}, 0\right) \oplus\left(0, \frac{1}{2}\right)$ & $N_{f}$ & $q_{\Psi}$ & $\bigotimes_{\alpha} R_{\Psi}^{\alpha}$ \\
$\chi$ & $\left(\frac{1}{2}, 0\right)$ & 1 & $q_{\chi}$ & $\bigotimes_{\alpha} R_{\chi}^{\alpha}$ \\
$\xi$ & $\left(\frac{1}{2}, 0\right)$ & 1 & $q_{\xi}$ & $\bigotimes_{\alpha} R_{\xi}^{\alpha}$ \\
$\phi$ & $(0,0)$ & 1 & $q_{\phi}$ & $\bigotimes_{\alpha} R_{\phi}^{\alpha}$ \\
\hline \hline
\end{tabular}

while the constraint on the Abelian charges reads

$$
q_{\phi}+q_{\chi}+q_{\xi}=0 .
$$

To prepare for the large-number-of-flavors limit, the gauge couplings for each gauge group $G_{\alpha}$ are rescaled as follows:

$$
K_{\alpha}=\frac{g_{\alpha}^{2} \mathcal{N} S_{2}\left(R_{\Psi}^{\alpha}\right)}{4 \pi^{2} d\left(R_{\Psi}^{\alpha}\right)}, \quad \text { where } \mathcal{N}=N_{f} \prod_{\alpha} d\left(R_{\Psi}^{\alpha}\right)
$$

Here the Dynkin index $S_{2}\left(R_{\Psi}^{\alpha}\right)$ is defined via the relation $S_{2}\left(R_{\Psi}^{\alpha}\right) \delta^{A B}=\operatorname{Tr}\left[T_{R_{\Psi}^{\alpha}}^{A} T_{R_{\Psi}^{\alpha}}^{B}\right.$. In the fundamental representation of an $\mathrm{SU}(N)$ group we take it to assume the value $1 / 2$. The dimension of a given representation is indicated with $d\left(R_{\Psi}^{\alpha}\right)$.

\section{A. Renormalization conventions}

We now briefly summarize our renormalization conventions to prepare for the computations of the renormalization group (RG) functions in the model. We denote all bare fields and couplings with subscript 0 .

In the Lagrangian (2.1), the bare fields renormalize according to

$$
\begin{aligned}
A_{\alpha, 0}^{\mu} & =Z_{A_{\alpha}}^{1 / 2} \mu^{-\epsilon / 2} A_{\alpha}^{\mu}, & & \Psi_{0}=Z_{\Psi}^{1 / 2} \mu^{-\epsilon / 2} \Psi, \\
\phi_{0} & =Z_{\phi}^{1 / 2} \mu^{-\epsilon / 2} \phi, & & \chi_{0}=Z_{\chi}^{1 / 2} \mu^{-\epsilon / 2} \chi, \\
\xi_{0} & =Z_{\xi}^{1 / 2} \mu^{-\epsilon / 2} \xi, & &
\end{aligned}
$$

while the bare couplings are given by

$$
\begin{aligned}
y_{0, a i j} & =\left(Z_{\chi} Z_{\xi} Z_{\phi}\right)^{-1 / 2} \mu^{\epsilon / 2}\left(y_{a i j}+\delta y_{a i j}\right), \\
\lambda_{0}^{a b}{ }_{c d} & =Z_{\phi}^{-2} \mu^{\epsilon}\left(\lambda^{a b}{ }_{c d}+\delta \lambda^{a b}{ }_{c d}\right), \\
g_{\alpha, 0} & =\tilde{g}_{\alpha, 0} \mu^{\epsilon / 2}=Z_{K_{\alpha}}^{-1 / 2} \mu^{\epsilon / 2} g_{\alpha} .
\end{aligned}
$$

We use dimensional regularization with $d=4-\epsilon$. The field renormalizations are expanded in terms of their $\epsilon$ poles, writing 


$$
Z_{i}=1+\sum_{k=1}^{\infty} \frac{1}{\epsilon^{k}} Z_{i}^{(k)}
$$

Similarly, the counterterms are expressed as

$\delta y_{a i j}=\sum_{k=1}^{\infty} \frac{1}{\epsilon^{k}} \delta y_{a i j}^{(k)}$ and $\delta \lambda^{a b}{ }_{c d}=\sum_{k=1}^{\infty} \frac{1}{\epsilon^{k}} \delta \lambda^{(k) a b}{ }_{c d}$.

It is now possible to expresses the beta function for the couplings in terms of the field-strength renormalizations and the counterterms of the renormalized Lagrangian in the above notation. The beta functions, $\beta_{x}=\mathrm{d} x / \mathrm{d} \ln \mu$, are given by

$$
\begin{aligned}
\beta_{y, a i j}= & \left(-\frac{1}{2}+K_{\beta} \frac{\partial}{\partial K_{\beta}}+\frac{y_{e k l}}{2} \frac{\partial}{\partial y_{e k l}}+\lambda^{e f}{ }_{g h} \frac{\partial}{\partial \lambda^{e f}{ }_{g h}}\right) \\
& \times\left[\delta y_{a i j}^{(1)}-\frac{Z_{\chi}^{(1)}+Z_{\xi}^{(1)}+Z_{\phi}^{(1)}}{2} y_{a i j}\right], \\
\beta_{\lambda^{a b} c d}= & \left(-1+K_{\beta} \frac{\partial}{\partial K_{\beta}}+\frac{y_{e k l}}{2} \frac{\partial}{\partial y_{e k l}}+\lambda^{e f}{ }_{g h} \frac{\partial}{\partial \lambda^{e f}}\right) \\
& \times\left[\delta \lambda^{(1) a b}{ }_{c d}-2 Z_{\phi}^{(1)} \lambda^{a b}{ }_{c d}\right], \\
\beta_{K_{\alpha}}= & \left(-1+K_{\beta} \frac{\partial}{\partial K_{\beta}}+\frac{y_{e k l}}{2} \frac{\partial}{\partial y_{e k l}}+\lambda^{e f}{ }_{g h} \frac{\partial}{\partial \lambda^{e f}{ }_{g h}}\right) \\
& \times\left[-Z_{K_{\alpha}}^{(1)} K_{\alpha}\right] .
\end{aligned}
$$

In order to practically evaluate the gauge field renormalization we make use of

$$
0=\operatorname{div}_{\epsilon}\left[Z_{A}\left(1-\Pi_{B}\left(\left\{x_{0}\right\}\right)\right)\right],
$$

where $\left\{x_{0}\right\}$ represents the full set of bare couplings and $\Pi_{B}$ is the bare, 1PI, 2-point function of the gauge bosons after having factorized out momenta and polarization structure; $i \Pi_{B, \mu \nu}(p)=i p^{2} \Delta_{\mu \nu}(p) \Pi_{B}\left(p^{2}\right)$ with $\Delta_{\mu \nu}(p)=$ $\eta_{\mu \nu}-p_{\mu} p_{\nu} / p^{2}$. Similarly, to compute the fermion and scalar field renormalization, we rely on the following relations involving the bare, 1PI, 2-point fermion, $-i \Sigma_{B}(p)$ and 2-point scalar, $-i S_{B}\left(p^{2}\right)$, functions,

$$
\begin{aligned}
& 0=\operatorname{div}_{\epsilon}\left[Z_{\chi, \xi}\left(1-\frac{\mathrm{d}}{\mathrm{d} \bar{\sigma} \cdot p} \Sigma_{\chi(\xi), B}\left(\left\{x_{0}\right\}\right)\right],\right. \\
& 0=\operatorname{div}_{\epsilon}\left[Z_{\phi}\left(1-\frac{\mathrm{d}}{\mathrm{d} p^{2}} S_{B}\left(\left\{x_{0}\right\}\right)\right)\right] .
\end{aligned}
$$

Finally for the renormalization of the couplings we employ

$$
\begin{aligned}
& 0=\operatorname{div}_{\epsilon}\left[Z_{\phi}^{1 / 2} Z_{\chi}^{1 / 2} Z_{\xi}^{1 / 2} Y_{B}\left(\left\{x_{0}\right\}\right)\right], \\
& 0=\operatorname{div}_{\epsilon}\left[Z_{\phi}^{2} \Lambda_{B}\left(\left\{x_{0}\right\}\right)\right],
\end{aligned}
$$

where $i Y_{B}, i \Lambda_{B}$ are the bare, 1PI, 3- and four-point functions. These are used to renormalize the Yukawa and quartic couplings.

\section{GAUGE-FERMION THEORY}

We start with reviewing the large $\mathcal{N}$ dynamics in gaugefermion theory investigated some time ago in [27-31]. This means that we drop $\phi, \chi$, and $\xi$ from the beginning. We extend the analysis to include semisimple gauge groups. The full dynamics including $\phi, \chi$, and $\xi$ is investigated in Sec. IV.

Only a limited set of diagrams contribute when computing the RG functions in the large $\mathcal{N}$ limit. In general the order, $(1 / \mathcal{N})^{k}$, of a diagram in the large $\mathcal{N}$ expansion can be determined as

$$
k=\text { powers of } g_{0}^{2}-\text { No. of fermion loops. }
$$

It follows that dressing gauge lines with $\Psi$ fermion bubbles (a bubble chain) does not increase the order of a diagram. To obtain the contribution at a given order in $1 / \mathcal{N}$, it is sufficient to consider a small set of diagrams, but one has to sum over the number of bubbles inserted on each gauge line. The resulting power series in $K$ is so well behaved that it is often possible to obtain a closed form expression for the $1 / \epsilon$ pole.

In the following computations we need to have an expression for the bubble chain. Each elementary bubble stems from a bare, 1PI, $\Psi$-fermion loop that in $\overline{\mathrm{MS}}$ reads

$$
\begin{aligned}
i \Pi_{\mu \nu}(p) & =i p^{2} \Delta_{\mu \nu}(p) \Pi_{0}\left(p^{2}\right), \quad \text { where } \\
\Pi_{0}\left(p^{2}\right) & =-2 K_{0} \Gamma_{0}(\epsilon)\left(-\frac{4 \pi \mu^{2}}{p^{2}}\right)^{\epsilon / 2} \text { and } \\
\Gamma_{0}(\epsilon) & =\frac{\Gamma^{2}\left(2-\frac{\epsilon}{2}\right) \Gamma\left(\frac{\epsilon}{2}\right)}{\Gamma(4-\epsilon)} .
\end{aligned}
$$

Note that $K_{0}$ is related to $\tilde{g}_{0}$ like $K_{\alpha}$ is related to $g_{\alpha}$ in (2.5). To avoid making the notation heavy we dropped a tilde on $K_{0}$.

The expression for a bubble chain with $n>0$ bubbles and $n+1$ free gauge propagators, $D_{\mu \nu}(p)$, reduces to

$$
\begin{aligned}
D_{\mu \nu}^{(n)}(p)= & {\left[D_{\mu \mu_{1}}(p) i \Pi^{\mu_{1} \mu_{2}}(p)\right] } \\
& \times\left[D_{\mu_{2} \mu_{3}}(p) i \Pi^{\mu_{3} \mu_{4}}(p)\right] \ldots D_{\mu_{2 n} \nu}(p) \\
= & \frac{-i}{p^{2}} \Delta_{\mu \nu}(p) \Pi_{0}^{n}\left(p^{2}\right) .
\end{aligned}
$$

The chain is fully transverse in $p$ because the gauge-fixing parameter does not renormalize in MS. In our computations we work in the Landau (Lorenz) gauge. This has the added benefit that $D_{\mu \nu}^{(n=0)}(p)=D_{\mu \nu}(p)$. The discussion above applies to each individual gauge group $\alpha$.

\section{A. Large $\mathcal{N}$ gauge beta function}

To determine the gauge beta function one has to compute the divergent part of the 2-point function. The leading order (LO) contribution in $1 / \mathcal{N}$ is simply given by one $\Psi$ bubble. The next-to-leading order (NLO) contribution, on the other 


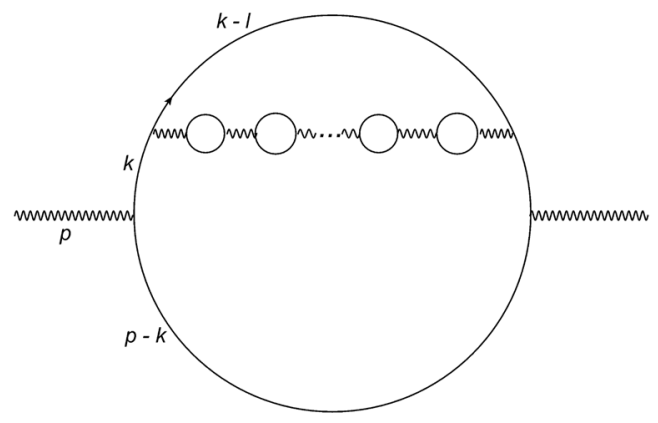

(a)

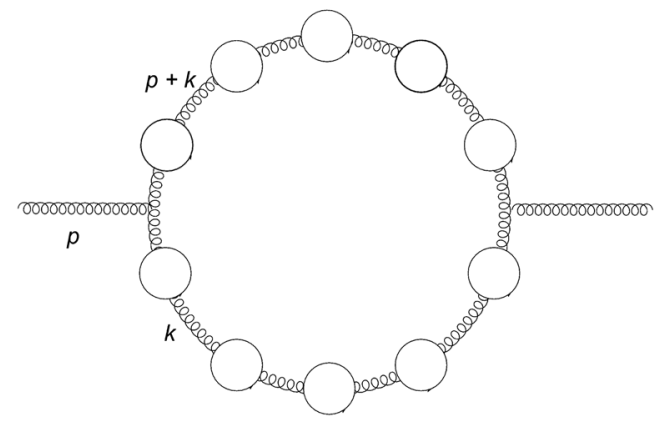

(c)

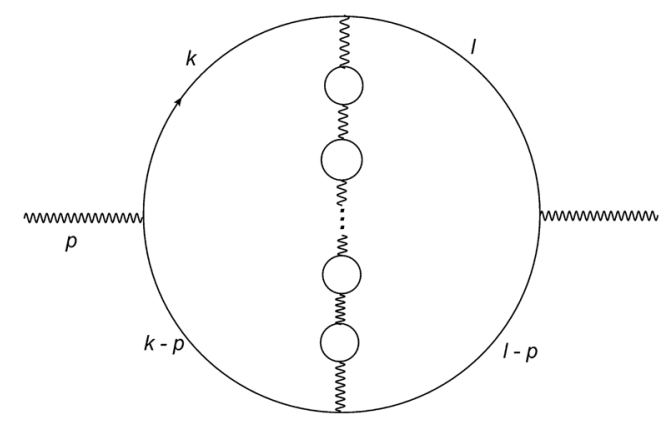

(b)

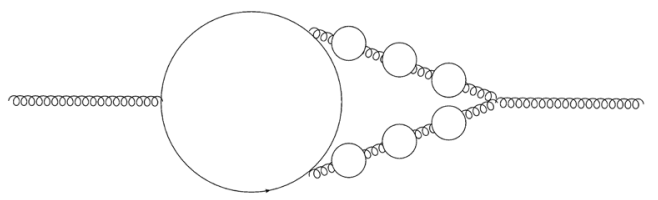

(d)

FIG. 1. Feynman diagrams for gauge field renormalization at order $1 / \mathcal{N}$. Diagrams (a) and (b) are present in both the Abelian and nonAbelian 2-point functions, while (c) and (d) only exist in the non-Abelian theory.

hand, is nontrivial and was computed in $[27,28]$ by evaluating the diagrams shown in Fig. 1. The first two diagrams of Figs. 1(a) and 1(b) yield

$$
\begin{aligned}
i \delta^{A B} p^{2} \Delta^{\mu \nu}(p) \Pi_{P}^{(n)}(p) \\
=-\left(-i \tilde{g}_{0}\right)^{4} \operatorname{Tr}\left[T_{\Psi}^{A} T_{\Psi}^{C} T_{\Psi}^{C} T_{\Psi}^{B}\right] \mu^{2 \epsilon} \int \frac{\mathrm{d}^{d} k}{(2 \pi)^{d}} \frac{\mathrm{d}^{d} \ell}{(2 \pi)^{d}} D_{\rho \sigma}^{(n)}(\ell) \\
\quad \times \operatorname{Tr}\left[\gamma^{\mu} \frac{i \not k}{k^{2}} \gamma^{\rho} \frac{i(\not k-\not h)}{(k-\ell)^{2}} \gamma^{\sigma} \frac{i \not k}{k^{2}} \gamma^{\nu} \frac{i(\not p-\not k)}{(p-k)^{2}}\right]
\end{aligned}
$$

$$
\begin{aligned}
i \delta^{A B} p^{2} \Delta^{\mu \nu}(p) \Pi_{T}^{(n)}(p) \\
=-\left(-i \tilde{g}_{0}\right)^{4} \operatorname{Tr}\left[T_{\Psi}^{A} T_{\Psi}^{C} T_{\Psi}^{B} T_{\Psi}^{C}\right] \mu^{2 \epsilon} \int \frac{\mathrm{d}^{d} k}{(2 \pi)^{d}} \frac{\mathrm{d}^{d} \ell}{(2 \pi)^{d}} D_{\rho \sigma}^{(n)}(k-\ell) \\
\quad \times \operatorname{Tr}\left[\gamma^{\mu} \frac{i(\not k-\not p)}{(k-p)^{2}} \gamma^{\rho} \frac{i(\not \ell-\not p)}{(\ell-p)^{2}} \gamma^{\nu} \frac{i \not \ell}{\ell^{2}} \gamma^{\sigma} \frac{i \not k}{k^{2}}\right]
\end{aligned}
$$

where $n \geq 0$ is the number of bubbles in the chain. These diagrams are present for all gauge groups. ${ }^{3}$ For the purpose of summing the contributions from all $n$, it is useful to extract the coupling and group structure from the 2-point functions

\footnotetext{
${ }^{3}$ In the Abelian case one replaces the gauge generators with the fermion charges $q_{\Psi}$.
}

$$
\begin{aligned}
& \Pi_{P}^{(n)}=\frac{d(G)}{\mathcal{N}} K_{0}^{n+2} A_{P}^{(n)} \\
& \Pi_{T}^{(n)}=\frac{d(G)}{\mathcal{N}}\left(1-\frac{1}{2} \frac{C_{2}(G)}{C_{2}\left(R_{\Psi}\right)}\right) K_{0}^{n+2} A_{T}^{(n)}
\end{aligned}
$$

Here the functions $A_{P, T}^{(n)}$ contain the loop structure of the respective diagrams and $C_{2}\left(R_{\Psi}\right)$ is the quadratic Casimir of the representation $R_{\Psi}$.

Going to the non-Abelian group we have additional contributions from the gluon self-interactions, cf., Figs. 1(c) and 1(d). The coupling and group structure from their contribution to the 2-point function is parametrized by

$$
\Pi_{G}^{(n)}=\frac{d(G)}{\mathcal{N}} \frac{C_{2}(G)}{C_{2}\left(R_{\Psi}\right)} K_{0}^{n+1} A_{G}^{(n)}
$$

We now review the final results for the gauge beta functions for the Abelian and non-Abelian gauge groups.

\section{Abelian beta function}

We consider the case where the $\Psi$ fermions are charged under a single $\mathrm{U}(1)$ gauge group and determine the associated gauge beta function. In this case, we point out that the $K$ coupling from Eq. (2.5) reduces to $K=g^{2} q_{\Psi}^{2} N_{f} / 4 \pi^{2}$ that agrees with earlier literature. The resummation of the beta function was performed first by Palanques-Mestre and 
Pascual [27]. Including both the LO and the $1 / \mathcal{N}$ contributions to the 1PI 2-point function, they found ${ }^{4}$

$$
\begin{aligned}
Z_{K} \Pi_{B}= & Z_{K} \Pi_{0}\left(p^{2}\right)+Z_{K} \frac{K_{0}}{\mathcal{N}} \sum_{n=1}^{\infty} K_{0}^{n}\left(A_{T}^{(n-1)}+2 A_{P}^{(n-1)}\right) \\
= & -2 K\left(-\frac{4 \pi \mu^{2}}{p^{2}}\right)^{\epsilon / 2} \Gamma_{0}(\epsilon) \\
& +\frac{3 K}{4 \mathcal{N}} \sum_{n=1}^{\infty}\left(-\frac{2 K_{0}}{3}\right)^{n} \frac{1}{(n+1) \epsilon^{n}} \tilde{F}(n+1, \epsilon) .
\end{aligned}
$$

Notice that we have used the fact that for Abelian gauge theory $Z_{K}=Z_{A}$. The function $\tilde{F}$ encodes the diagram structure, and it turns out that the beta function depends only on $\tilde{F}(0, \epsilon)$. Using analyticity of $\tilde{F}$, we can apply the resummation formula (A4) to obtain

$Z_{K}^{(1)}=\left.Z_{K} \Pi_{B}\right|_{1 / \epsilon}=-\frac{2 K}{3}-\frac{1}{2 \mathcal{N}} \int_{0}^{K} \mathrm{~d} x(K-x) \tilde{F}\left(0, \frac{2}{3} x\right)$,

where

$$
\tilde{F}(0, x)=\frac{(1-x)\left(1-\frac{x}{3}\right)\left(1+\frac{x}{2}\right) \Gamma(4-x)}{3 \Gamma^{2}\left(2-\frac{x}{2}\right) \Gamma\left(3-\frac{x}{2}\right) \Gamma\left(1+\frac{x}{2}\right)} .
$$

Finally, applying (2.10) the gauge beta function reads

$$
\beta_{K}=-K^{2} \frac{\partial}{\partial K} Z_{K}^{(1)}=\frac{2 K^{2}}{3}\left[1+\frac{1}{\mathcal{N}} F_{1}(K)\right]
$$

to NLO in $1 / \mathcal{N}$. For later convenience we introduced

$$
F_{1}(K)=\frac{3}{4} \int_{0}^{K} \mathrm{~d} x \tilde{F}\left(0, \frac{2}{3} x\right) .
$$

\section{Non-Abelian beta function}

Now we turn to the case where the $\Psi$ fermions transform under a given representation $R_{\Psi}$ of a non-Abelian gauge group. In this instance, the gauge field 2-point function gets an additional contribution due to the gluon self-interaction. The NLO 2-point function is then given by

$$
\begin{aligned}
\Pi_{B}= & \Pi_{0}+K_{0} \frac{d(G)}{\mathcal{N}} \\
& \times \sum_{n=1}\left\{K_{0}^{n}\left[\left(1-\frac{C_{2}(G)}{2 C_{2}\left(R_{\Psi}\right)}\right) A_{T}^{(n-1)}+2 A_{P}^{(n-1)}\right]\right. \\
& \left.+K_{0}^{n-1} \frac{C_{2}(G)}{C_{2}\left(R_{\Psi}\right)} A_{G}^{(n-1)}\right\} .
\end{aligned}
$$

\footnotetext{
${ }^{4}$ The function $\tilde{F}$ is related to the Mestre-Pascual result via $\tilde{F}(n, \epsilon)=F_{\mathrm{MP}}(n,-\epsilon / 2)$.
}

In the non-Abelian case the gauge-coupling renormalization is more involved. The computation can either be performed in the background field gauge or in $\xi$ gauge provided that for the latter one includes the vertex renormalization. This computation was originally performed in [28] using an alternative method and later reviewed in [29]; and the result reads

$$
\beta_{K}=\frac{2 K^{2}}{3}\left[1+\frac{d(G)}{\mathcal{N}} H_{1}(K)\right],
$$

where we have defined the functions

$$
\begin{aligned}
H_{1}(K) & =-\frac{11 C_{2}(G)}{4 C_{2}\left(R_{\Psi}\right)}+\frac{3}{4} \int_{0}^{K} \mathrm{~d} x \tilde{F}\left(0, \frac{2}{3} x\right) \tilde{G}\left(\frac{1}{3} x\right), \\
\tilde{G}(x) & =1+\frac{C_{2}(G)}{C_{2}\left(R_{\Psi}\right)} \frac{20-43 x+32 x^{2}-14 x^{3}+4 x^{4}}{4(2 x-1)(2 x-3)\left(1-x^{2}\right)} .
\end{aligned}
$$

While $\tilde{F}$ is the function obtained in the $\mathrm{U}(1)$ case, the nontrivial part of $\tilde{G}$ stems from the gluon contribution. ${ }^{5}$ Notice that $H_{1}$ reduces to $F_{1}$ in the case of an Abelian gauge group, so Eq. (3.14) is valid for all simple gauge groups.

\section{B. Extension to semisimple gauge groups}

Let us now generalize the result to the case where the vectorlike fermions are charged under a semisimple gauge group. To determine the mixed contribution to the gaugecoupling renormalization $Z_{K_{\alpha}}$ it is sufficient to consider only the mixed diagrams appearing in the gauge 2-point function. In the $\xi$ gauges the mixed contributions to the vertex and fermion field renormalization cancel against each other. To determine $Z_{A_{\alpha}}$ we employ Eq. (2.11) to accommodate mixed gauge contributions. Starting from one gauge field, $\alpha$, the 2-point function contains the usual terms present in Eq. (3.13). These are unaffected by the presence of other gauge groups. Additionally, at NLO, it is possible to have a gauge bubble chain from a different gauge group $\beta$ stretching across the fermion loop instead of the original $\alpha$ chain, as shown in Fig. 2. These are the only type of diagrams mixing the gauge groups, since a single fermion bubble cannot couple simultaneously to two different gauge groups. The new contribution to the 1PI 2-point function coming from the mixed diagrams with the group $\beta$ is

$$
\begin{aligned}
Z_{A_{\alpha}} \Delta \Pi_{\alpha, B} & =Z_{A_{\alpha}} K_{\alpha, 0} \frac{d\left(G_{\beta}\right)}{\mathcal{N}} \sum_{n=1}^{\infty} K_{\beta, 0}^{n}\left(A_{T}^{(n-1)}+2 A_{P}^{(n-1)}\right) \\
& =\frac{3 d\left(G_{\beta}\right)}{4 \mathcal{N}} K_{\alpha} \sum_{n=1}^{\infty}\left(-\frac{2 K_{\beta, 0}}{3}\right)^{n} \frac{\tilde{F}(n+1, \epsilon)}{(n+1) \epsilon^{n}} .
\end{aligned}
$$

To renormalize $K_{\alpha, 0}$, we have used the fact that $Z_{K_{\alpha}}=Z_{A_{\alpha}}$ at LO in $1 / \mathcal{N}$. Once again, the $1 / \epsilon$ pole can be extracted using the resummation formula (A4). The new contribution to the gauge-coupling renormalization is obtained as

\footnotetext{
${ }^{5} \tilde{G}(x)=\frac{d\left(R_{\Psi}\right)}{d(G)} I_{2}(x)$ when comparing with the result in [29].
} 

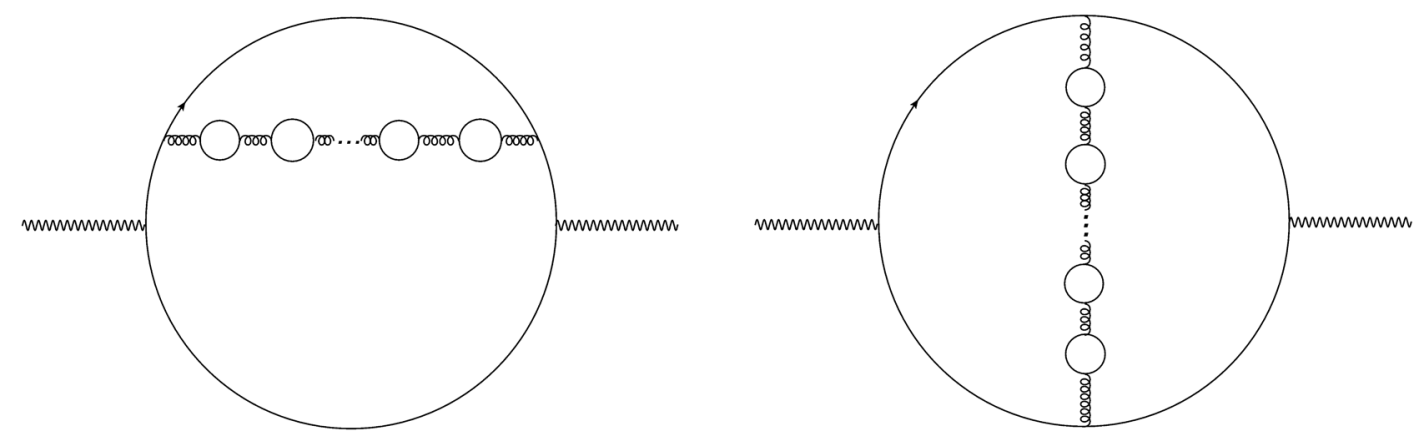

FIG. 2. Feynman diagrams for the 2-point functions giving mixed terms to the beta functions.

$\Delta Z_{K_{\alpha}}^{(1)}=\Delta Z_{A_{\alpha}}^{(1)}=-\frac{d\left(G_{\beta}\right)}{2 \mathcal{N}} K_{\alpha} \int_{0}^{K_{\beta}} \mathrm{d} x\left(1-\frac{x}{K_{\beta}}\right) \tilde{F}\left(0, \frac{2}{3} x\right) . \quad \beta_{K_{\alpha}}=\frac{2 K_{\alpha}^{2}}{3}\left[1+\frac{d\left(G_{\alpha}\right)}{\mathcal{N}} H_{1}^{(\alpha)}\left(K_{\alpha}\right)+\frac{1}{\mathcal{N}} \sum_{\beta \neq \alpha} d\left(G_{\beta}\right) F_{1}\left(K_{\beta}\right)\right]$.

The mixed contributions to the beta function read

$$
\begin{aligned}
\Delta \beta_{K_{\alpha}} & =-K_{\alpha}\left(K_{\alpha} \frac{\partial}{\partial K_{\alpha}}+K_{\beta} \frac{\partial}{\partial K_{\beta}}\right) \Delta Z_{K_{\alpha}}^{(1)} \\
& =\frac{d\left(G_{\beta}\right)}{2 \mathcal{N}} K_{\alpha}^{2} \int_{0}^{K_{\beta}} \mathrm{d} x \tilde{F}\left(0, \frac{2}{3} x\right) .
\end{aligned}
$$

Taking into account the mixed contributions coming from all the different gauge groups to each beta function we find
Note that the $H_{1}$ functions are dependent on the specific gauge group and fermion representation as evident from Eq. (3.15) (hence the superscript). In the case of an Abelian group $H_{1}$ reduces to $F_{1}$.

A test of our results consists in checking that when reexpanding the beta functions given in Eq. (3.19) as functions of the couplings the coefficients agree with the state-of-the-art three-loop perturbative computation [38] which for $G=G_{\alpha} \times G_{\beta}$ reads

$$
\begin{aligned}
\beta_{K_{\alpha}}^{\text {three-loop }}=\frac{2 K_{\alpha}^{2}}{3}[1 & +\frac{1}{\mathcal{N}}\left(\frac{K_{\alpha}\left(5 C_{2}\left(G_{\alpha}\right)+3 C_{2}\left(R_{\Psi}^{\alpha}\right)\right)}{4 S_{2}\left(R_{\Psi}^{\alpha}\right) d\left(R_{\Psi}^{\alpha}\right)^{-1}}-\frac{K_{\alpha}^{2}\left(79 C_{2}\left(G_{\alpha}\right)+66 C_{2}\left(R_{\Psi}^{\alpha}\right)\right)}{288 S_{2}\left(R_{\Psi}^{\alpha}\right) d\left(R_{\Psi}^{\alpha}\right)^{-1}}\right) \\
& +\frac{1}{\mathcal{N}}\left(\frac{3 K_{\beta} C_{2}\left(R_{\Psi}^{\beta}\right)}{4 S_{2}\left(R_{\Psi}^{\beta}\right) d\left(R_{\Psi}^{\beta}\right)^{-1}}-\frac{11 K_{\beta}^{2} C_{2}\left(R_{\Psi}^{\beta}\right)}{48 S\left(R_{\Psi}^{\beta}\right) d\left(R_{\Psi}^{\beta}\right)^{-1}}\right)-\frac{1}{\mathcal{N}^{2}}\left(\frac{17 K_{\alpha} C_{2}\left(G_{\alpha}\right)^{2}}{3 S_{2}\left(R_{\Psi}^{\alpha}\right)^{2} d\left(R_{\Psi}^{\alpha}\right)^{-2}}\right. \\
& \left.\left.+K_{\alpha}^{2} \frac{1415 C_{2}\left(G_{\alpha}\right)^{2}+615 C_{2}\left(G_{\alpha}\right) C_{2}\left(R_{\Psi}^{\alpha}\right)-288 C_{2}\left(R_{\Psi}^{\alpha}\right)^{2}}{288 S_{2}\left(R_{\Psi}^{\alpha}\right)^{2} d\left(R_{\Psi}^{\alpha}\right)^{-2}}+\cdots\right)-\frac{1}{\mathcal{N}^{3}}\left(K_{\alpha}^{2} \frac{2857 C_{2}\left(R_{\Psi}^{\alpha}\right)^{3}}{288 S_{2}\left(R_{\Psi}^{\alpha}\right)^{3} d\left(R_{\Psi}^{\alpha}\right)^{-3}}+\cdots\right)\right] .
\end{aligned}
$$

It is straightforward to check that the leading $1 / \mathcal{N}$ terms agree with the corresponding terms in Eq. (3.19).

In the derivation of Eq. (3.19) we have assumed that the gauge group under which $\Psi$ is charged contains at most one $\mathrm{U}(1)$. If that were not the case, it would be possible for the fermion bubbles to couple to two different Abelian groups simultaneously. This would give a new class of diagrams, where the bubble chains would alternate between the two groups. In such a case one would also have to take into account kinetic mixing between the two gauge groups. This has not been considered here.

\section{Safe phase diagrams}

To conclude this section, we investigate the short distance fate of gauge-fermion theories at a large number of matter fields. Here asymptotic freedom is lost and unless an interacting UV fixed point emerges, the underlying theory can be viewed, at best, as an effective low-energy description of physical phenomena. In this regime asymptotic safety is dynamically achieved due to the collective effect of the many fermions present in the theory. This is reflected in the emergence of a nontrivial 0 of the beta functions at NLO in $1 / \mathcal{N}$ [32].

\section{Safe $Q C D$}

For single gauge groups, resembling QCD with many flavors, asymptotic safety is indeed a possibility [32]. To elucidate this point while making this work self-contained, we briefly summarize here the salient points of how a UV fixed point emerges. To make our point clear, we consider 


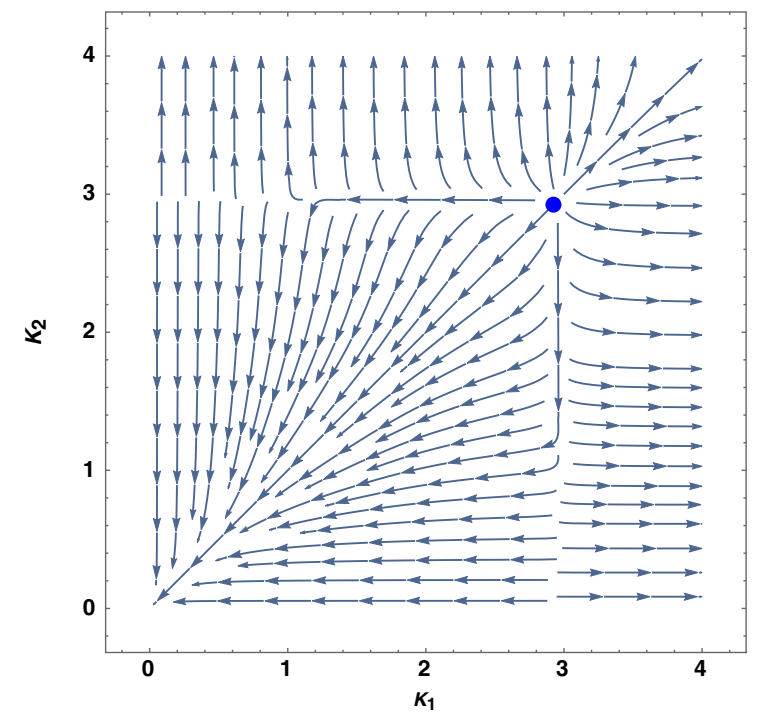

(a) $\mathrm{SU}\left(N_{1}\right) \times \mathrm{SU}\left(N_{2}\right)$

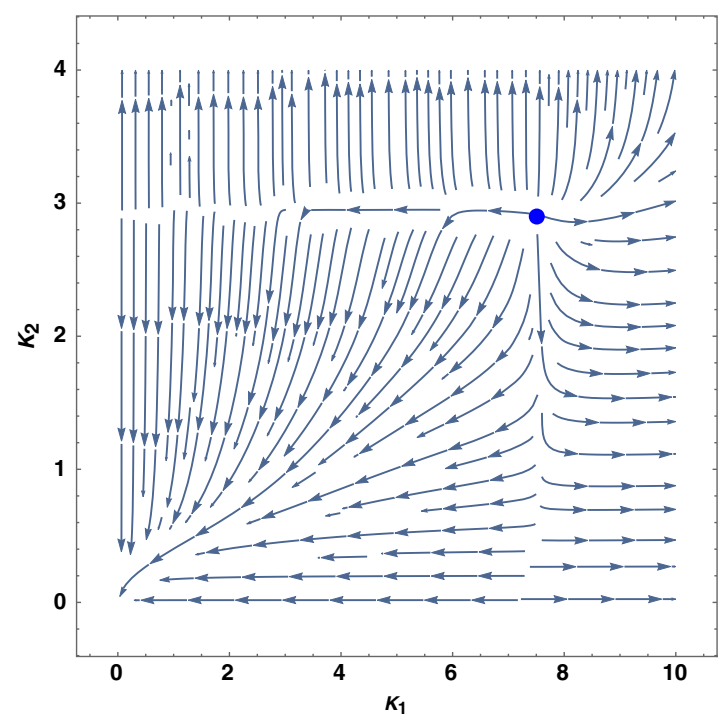

(b) $\mathrm{U}(1) \times \mathrm{SU}(N)$

FIG. 3. Phase diagrams of semisimple gauge theories consisting of two non-Abelian groups (left) and an Abelian and a non-Abelian group (right).

an $\mathrm{SU}(N)$ gauge group with $N_{f}$ flavors transforming according to the fundamental representation. From Eq. (3.14), one shows that there is a fixed point a $K^{*}=3$ up to exponentially vanishing corrections [32]. This occurs because the $\tilde{G}(x)$ function in (3.15) has a pole in the integrand at $x=1(K=3)$, corresponding to a logarithmic singularity in the $H_{1}(K)$ function. The beta function therefore has a UV fixed point at $K^{*} \approx 3$ to leading order in $1 / \mathcal{N}$, which is obtained from the condition $1+d(G) H_{1}(K) / \mathcal{N}=0$. The new conformal window for these theories as a function of the number of flavors and colors extends the original infrared (IR) conformal window to also contain the asymptotically safe scenario [32]. It is worth mentioning that to provide a rough estimate of the lower boundary of the asymptotically safe window, one can use the stability of the $1 / \mathcal{N}$ expansion $[29,32]$ by estimating when the $1 / \mathcal{N}^{2}$ and higher corrections become relevant.

\section{Safe semisimple gauge groups}

We now investigate the semisimple case starting with the $G=\mathrm{SU}\left(N_{1}\right) \times \mathrm{SU}\left(N_{2}\right)$ example. The associated phase diagram refers to the RG flow plotted in the plane of the two gauge couplings, $K_{1}$ and $K_{2}$, and it is presented in Fig. 3(a). The UV interacting fixed point, repulsive in all directions, occurs for $K_{1}^{*}=K_{2}^{*}=3$ (the blue-dot) up to exponentially small corrections. Two more interacting fixed points occur for $\left(K_{1}^{*}=3, K_{2}^{*}=0\right)$ and $\left(K_{1}^{*}=0, K_{2}^{*}=3\right)$ corresponding to the fixed points of each single gauge group. Finally we have the Gaussian IR fixed point at the origin of the coupling space. This analysis complements the perturbative analysis for semisimple gauge groups investigated first in [39]. We therefore discover that there is a UV complete fixed point for semisimple gauge theories with a two-dimensional critical surface.
The phase diagram for the semisimple group $G=$ $\mathrm{U}(1) \times \mathrm{SU}\left(N_{2}\right)$ is presented in Fig. 3(b). It is structurally identical to the $\operatorname{SU}\left(N_{1}\right) \times \operatorname{SU}\left(N_{2}\right)$ case above with the difference that the UV fixed point for the U(1) gauge couplings occurs at $K_{1}^{*}=15 / 2$.

One can derive a rough estimate of the asymptotically safe conformal window for the semisimple gauge group as well. We use again the stability of the $1 / \mathcal{N}$ expansion by estimating numerically the size of the known $1 / \mathcal{N}^{2}$ and $1 / \mathcal{N}^{3}$ corrections from Eq. (3.20). We expect it to be wider than safe QCD because the effective number of flavors $\mathcal{N}=N_{f} d\left(R_{\Psi}^{1}\right) d\left(R_{\Psi}^{2}\right)$ is larger.

\section{YUKAWA AND SELF-COUPLING BETA FUNCTIONS}

We now review and further elucidate the computation of the RG functions of the Yukawa [35] and quartic couplings [14] of the model (2.1) in the presence of a large number of vectorlike fermions. Finally, the results of the running of the quartic coupling are extended to the case where the $\Psi$ fermions transform under a semisimple gauge group. We are interested in the case in which $y$ and $\lambda$ scale with $\mathcal{N}$ as $\lambda \sim y^{2} \sim 1 / \mathcal{N}$. This is the region for which a UV fixed point can appear due to the interplay between the large $\mathcal{N}$ gauge contribution and leading corrections stemming from the Yukawa and scalar self-coupling. With this scaling of the couplings it is sufficient to consider the one-loop contributions from the Yukawa and quartic coupling to their beta functions. The counting then ensures that higher loops give corrections that are higher order in $1 / \mathcal{N}$.

The leading $1 / \mathcal{N}$ contribution stemming from the $\Psi$ fermions is obtained by dressing gauge lines with their 


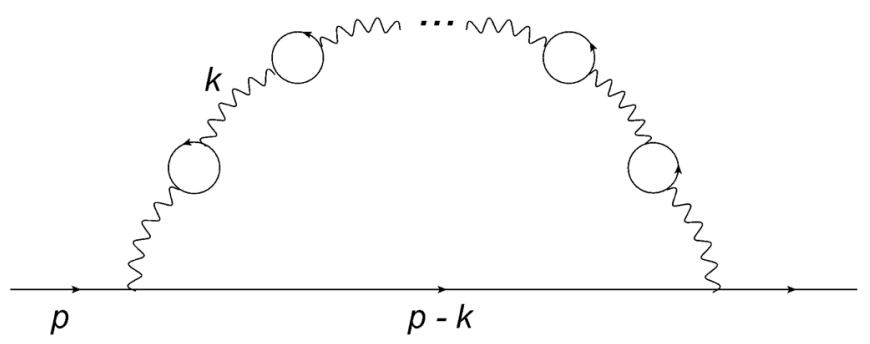

FIG. 4. LO gauge contribution to the fermion self-energy.

bubbles. As in Sec. III, these diagrams can be resummed and the $1 / \epsilon$ pole extracted in a closed from. We first discuss the new contribution to the fermion and scalar self-energies before moving to compute the vertex corrections. We see that it is straightforward to generalize the results to the semisimple case except for the quartic self-coupling.

\section{A. Fermion self-energy}

To compute the Yukawa beta function we need first to compute the gauge correction to the fermion self-energy to LO in $1 / \mathcal{N}$ for the $\chi, \xi$ fermions.

\section{Abelian case}

We start with the Abelian case and then extend the result to the non-Abelian one. At this order in $1 / \mathcal{N}$ the relevant diagram is shown in Fig. 4. For the $\chi$ (identically for $\xi$ ) selfenergy, the defining integral for the $n$-bubble diagram is

$-i \Sigma_{\chi}^{(n)}(p)=\left(i q_{\chi} \tilde{g}_{0}\right)^{2} \mu^{\epsilon} \int \frac{\mathrm{d}^{d} k}{(2 \pi)^{d}} \bar{\sigma}^{\mu} \frac{i \sigma \cdot(p-k)}{(p-k)^{2}} \bar{\sigma}^{\nu} D_{\nu \mu}^{(n)}(k)$.

The integral is known, and the diagram evaluates to

$$
\begin{aligned}
-i \Sigma_{\chi}^{(n)}(p)= & -\frac{3 i}{8 \mathcal{N}} \frac{q_{\chi}^{2}}{q_{\Psi}^{2}} \bar{\sigma} \cdot p\left(-\frac{2 K_{0}}{3}\right)^{n+1} 3^{n} \Gamma_{0}^{n}(\epsilon) \Gamma_{\psi}(n, \epsilon) \\
& \times\left(-\frac{4 \pi \mu^{2}}{p^{2}}\right)^{(n+1) \epsilon / 2},
\end{aligned}
$$

where we defined

$\Gamma_{\psi}(n, \epsilon)=\frac{n(3-\epsilon)}{n+1} \frac{\Gamma\left(2-\frac{\epsilon}{2}\right) \Gamma\left(1+\frac{n+1}{2} \epsilon\right) \Gamma\left(1-\frac{n+1}{2} \epsilon\right)}{\Gamma\left(2+\frac{n}{2} \epsilon\right) \Gamma\left(3-\frac{n+2}{2} \epsilon\right)}$.

Summing over all bubbles to obtain the total gauge contribution to the self-energy at $1 / \mathcal{N}$ and shifting the sum from $n \rightarrow n-1$, we obtain

$$
\frac{\mathrm{d} \Sigma_{\chi}}{\mathrm{d} \bar{\sigma} \cdot p}=-\frac{9}{16 \mathcal{N}} \frac{q_{\chi}^{2}}{q_{\Psi}^{2}} \sum_{n=1}^{\infty}\left(-\frac{2 K_{0}}{3}\right)^{n} \frac{1}{n \epsilon^{n}} H_{\Psi}(n, \epsilon),
$$

where

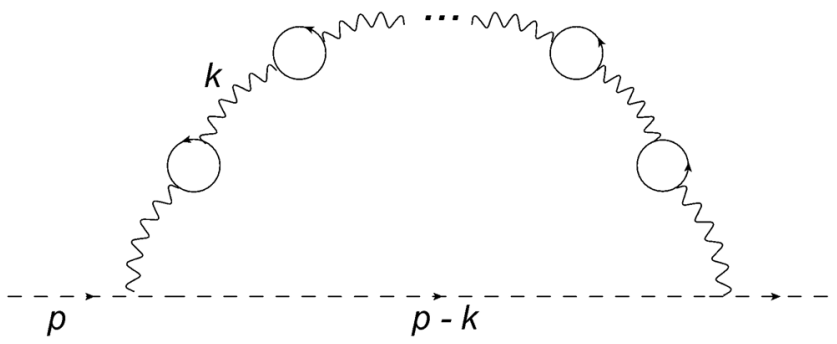

FIG. 5. LO gauge contribution to the scalar self-energy.

$$
\begin{aligned}
H_{\psi}(n, \epsilon)= & -\frac{2}{3}\left(-\frac{4 \pi \mu^{2}}{p^{2}}\right)^{n \epsilon / 2} \\
& \times\left[3 \epsilon \Gamma_{0}(\epsilon)\right]^{n-1}(1-n \epsilon) n \epsilon \Gamma_{\psi}(n-1, \epsilon) .
\end{aligned}
$$

The contribution to the RG function stems from the $1 / \epsilon$ pole which is extracted using the resummation formula (A15) and yields

$Z_{\chi(\xi)}^{(1)}=\left.\frac{\mathrm{d} \Sigma_{\chi(\xi)}}{\mathrm{d} \bar{\sigma} \cdot p}\right|_{1 / \epsilon}=\frac{3}{8 \mathcal{N}} \frac{q_{\chi(\xi)}^{2}}{q_{\Psi}^{2}} \int_{0}^{K} \mathrm{~d} x H_{\psi}\left(0, \frac{2}{3} x\right)$.

To arrive at the above relation between $Z_{\chi}$ and the 2-point function we used the fact that $Z_{\chi}=1+\mathcal{O}(1 / \mathcal{N})$. For the reader's convenience we also give the expression for $H_{\psi}$,

$$
H_{\psi}(0, x)=\frac{x\left(1-\frac{x}{3}\right) \Gamma(4-x)}{3 \Gamma^{2}(2-x) \Gamma\left(3-\frac{x}{2}\right) \Gamma\left(1+\frac{x}{2}\right)} .
$$

\section{Non-Abelian case}

The result for the non-Abelian gauge group case is obtained by replacing

$\left(q_{\chi} \tilde{g}_{0}\right)^{2} \rightarrow \tilde{g}_{0}^{2}\left(T_{\chi}^{A} T_{\chi}^{A}\right)^{i}{ }_{j}=\frac{4 \pi^{2} d\left(R_{\Psi}\right) K_{0}}{\mathcal{N} S_{2}\left(R_{\Psi}\right)} C_{2}\left(R_{\chi}\right) \delta_{j}^{i}$

in the $n$-bubble self-energy (4.1). The rest of the computation follows the Abelian case yielding the field-strength renormalization

$Z_{\chi(\xi)}^{(1)}=\frac{3 d\left(R_{\Psi}\right)}{8 \mathcal{N} S_{2}\left(R_{\Psi}\right)} C_{2}\left(R_{\chi(\xi)}\right) \int_{0}^{K} \mathrm{~d} x H_{\psi}\left(0, \frac{2}{3} x\right)$.

\section{B. Scalar self-energy}

We proceed to determine the correction to the scalar selfenergy at LO in $1 / \mathcal{N}$. This is a necessary step towards the full computation of the Yukawa and quartic self-coupling.

\section{Abelian case}

Here the diagrams that contribute contain a chain of $n$ fermion bubbles as shown in Fig. 5. Analytically 


$$
\begin{aligned}
-i S^{(n)}\left(p^{2}\right)= & \left(i \tilde{g}_{0} q_{\phi}\right)^{2} \mu^{\epsilon} \int \frac{\mathrm{d}^{d} k}{(2 \pi)^{d}} \frac{i}{(p-k)^{2}}(2 p-k)^{\mu} D_{\mu \nu}^{(n)}(k) \\
& \times(2 p-k)^{\nu} .
\end{aligned}
$$

The integral yields the $n$-bubble contribution

$$
\begin{aligned}
-i S^{(n)}\left(p^{2}\right)= & -i \frac{K_{0} q_{\phi}^{2}}{\mathcal{N} q_{\Psi}^{2}}\left(-2 K_{0}\right)^{n} p^{2}\left(-\frac{4 \pi \mu^{2}}{p^{2}}\right)^{(n+1) \epsilon / 2} \\
& \times(3-\epsilon) \Gamma_{0}^{n}(\epsilon) \Gamma_{\phi}(n, \epsilon),
\end{aligned}
$$

where we have defined the function

$$
\Gamma_{\phi}(n, \epsilon)=\frac{\Gamma\left(1-\frac{n+1}{2} \epsilon\right) \Gamma\left(2-\frac{\epsilon}{2}\right) \Gamma\left(\frac{n+1}{2} \epsilon\right)}{2 \Gamma\left(2+\frac{n}{2} \epsilon\right) \Gamma\left(3-\frac{n+2}{2} \epsilon\right)} .
$$

Summing over $S^{(n)}\left(p^{2}\right)$ and shifting the summation from $n$ to $n-1$, one can rewrite the derivative with respect to $p^{2}$ in the form

$$
\frac{\mathrm{d}}{\mathrm{d} p^{2}} S\left(p^{2}\right)=-\frac{9 q_{\phi}^{2}}{4 \mathcal{N} q_{\Psi}^{2}} \sum_{n=1}^{\infty}\left(-\frac{2 K_{0}}{3}\right)^{n} \frac{1}{n \epsilon^{n}} H_{\phi}(n, \epsilon) .
$$

Here we defined

$$
\begin{aligned}
H_{\phi}(n, \epsilon)= & 4\left(-\frac{4 \pi \mu^{2}}{p^{2}}\right)^{n \epsilon / 2}\left(1-\frac{n}{2} \epsilon\right)\left(1-\frac{\epsilon}{3}\right) \\
& \times\left[3 \epsilon \Gamma_{0}(\epsilon)\right]^{n-1} \Gamma_{\phi}(n-1, \epsilon) .
\end{aligned}
$$

The simple $\epsilon$ pole of interest for the RG function is determined using (A15) and it yields

$Z_{\phi}^{(1)}=\left.\frac{\mathrm{d}}{\mathrm{d} p^{2}} S\left(p^{2}\right)\right|_{1 / \epsilon}=\frac{3 q_{\phi}^{2}}{2 \mathcal{N} q_{\Psi}^{2}} \int_{0}^{K} \mathrm{~d} x H_{\phi}\left(0, \frac{2}{3} x\right)$

where

$$
H_{\phi}(0, x)=\frac{\left(1-\frac{x}{3}\right) \Gamma(4-x)}{3 \Gamma^{2}\left(2-\frac{x}{2}\right) \Gamma\left(3-\frac{x}{2}\right) \Gamma\left(1+\frac{x}{2}\right)} .
$$

\section{Non-Abelian case}

To determine the scalar self-energy for the non-Abelian case one replaces the $\mathrm{U}(1)$ charges in (4.10) as follows:

$$
\left(q_{\phi} \tilde{g}_{0}\right)^{2} \rightarrow \tilde{g}_{0}^{2}\left(T_{\phi}^{A} T_{\phi}^{A}\right)^{a}{ }_{b}=\frac{4 \pi^{2} d\left(R_{\Psi}\right) K_{0}}{\mathcal{N} S_{2}\left(R_{\Psi}\right)} C_{2}\left(R_{\phi}\right) \delta^{a}{ }_{b}
$$

The rest of the computation is identical to the Abelian case and yields

$$
Z_{\phi}^{(1)}=\frac{3 d\left(R_{\Psi}\right)}{2 \mathcal{N} S_{2}\left(R_{\Psi}\right)} C_{2}\left(R_{\phi}\right) \int_{0}^{K} \mathrm{~d} x H_{\phi}\left(0, \frac{2}{3} x\right) .
$$

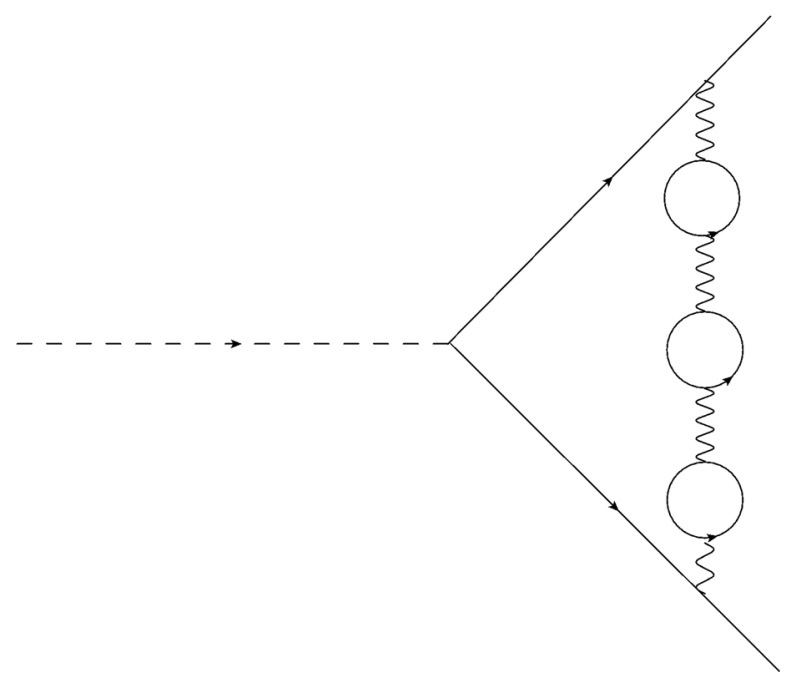

FIG. 6. Contributions to the Yukawa vertex at order $1 / \mathcal{N}$.

\section{Yukawa vertex}

The only vertex diagram that contributes to the Yukawa beta function in the Landau gauge is shown in Fig. 6. The other diagrams vanish trivially in this gauge when the external momenta are set to 0 .

\section{Abelian case}

With vanishing external momenta, the analytic expression representing the diagrams contributing to the Yukawa coupling with $n$ bubbles on the gauge line is

$$
\begin{aligned}
i Y^{(n)}= & (-i y)\left(-i q_{\chi} \tilde{g}_{0}\right)\left(i q_{\xi} \tilde{g}_{0}\right) \mu^{\epsilon} \\
& \times \int \frac{\mathrm{d}^{d} k}{(2 \pi)^{d}} \sigma^{\mu} \frac{i \bar{\sigma} \cdot k}{k^{2}-m^{2}} \frac{i \sigma \cdot k}{k^{2}-m^{2}} \bar{\sigma}^{\nu} D_{\mu \nu}^{(n)}(k) .
\end{aligned}
$$

A common mass has been added to the fermion propagators as an IR regulator, as it does not influence the divergent part of the diagram. One finds that

$$
\begin{aligned}
i Y^{(n)}= & -\frac{i 3 y}{8 \mathcal{N}} \frac{q_{\chi} q_{\xi}}{q_{\Psi}^{2}}\left(-2 K_{0}\right)^{n+1} \Gamma_{0}(\epsilon)^{n}\left(\frac{4 \pi \mu^{2}}{m^{2}}\right)^{(n+1) \epsilon / 2} \\
& \times\left(1-\frac{\epsilon}{3}\right) \frac{\Gamma\left(2-\frac{n+1}{2} \epsilon\right) \Gamma\left(\frac{n+1}{2} \epsilon\right)}{\Gamma\left(2-\frac{\epsilon}{2}\right)}
\end{aligned}
$$

Next we sum over every number of fermion bubbles to find the full $1 / \mathcal{N}$ gauge contribution to the vertex, and cast the expression in a suitable form, yielding

$$
i Y=-i y \frac{9}{4 \mathcal{N}} \frac{q_{\chi} q_{\xi}}{q_{\Psi}^{2}} \sum_{n=1}^{\infty}\left(-\frac{2 K_{0}}{3}\right)^{n} \frac{1}{n \epsilon^{n}} H_{y}(n, \epsilon),
$$

where 


$$
\begin{aligned}
H_{y}(n, \epsilon)= & \left(\frac{4 \pi \mu^{2}}{m^{2}}\right)^{n \epsilon / 2}\left[3 \epsilon \Gamma_{0}(\epsilon)\right]^{n-1} \\
& \times\left(1-\frac{\epsilon}{3}\right) \frac{\Gamma\left(2-\frac{n}{2} \epsilon\right) \Gamma\left(1+\frac{n}{2} \epsilon\right)}{\Gamma\left(2-\frac{\epsilon}{2}\right)} .
\end{aligned}
$$

Following the usual procedure the $1 / \epsilon$ pole can be extracted in a closed form using (A15), as $H_{y}$ is sufficiently regular. The counterterm for the Yukawa coupling is then extracted via

$$
\delta y^{(1)}=\left.Y\right|_{1 / \epsilon}=y \frac{3}{2 \mathcal{N}} \frac{q_{\chi} q_{\xi}}{q_{\Psi}^{2}} \int_{0}^{K} \mathrm{~d} x H_{y}\left(0, \frac{2}{3} x\right)
$$

with

$$
H_{y}(0, x)=\frac{\left(1-\frac{x}{3}\right) \Gamma(4-x)}{6 \Gamma^{3}\left(2-\frac{x}{2}\right) \Gamma\left(1+\frac{x}{2}\right)}
$$

\section{Non-Abelian case}

The previous result can be extended to the non-Abelian case provided that we use

$$
\begin{aligned}
y q_{\chi} q_{\xi} \tilde{g}_{0}^{2} & \rightarrow y_{a k l} \tilde{g}_{0}^{2}\left(T_{\chi}^{A}\right)^{k}{ }_{i}\left(T_{\xi}^{A}\right)^{l}{ }_{j} \\
& =y_{a k l}\left(T_{\chi}^{A}\right)^{k}{ }_{i}\left(T_{\xi}^{A}\right)^{l}{ }_{j} \frac{4 \pi^{2} d\left(R_{\Psi}\right) K_{0}}{\mathcal{N} S_{2}\left(R_{\Psi}\right)}
\end{aligned}
$$

in the Abelian expression (4.19). Employing the identity

$y_{a k l}\left(T_{\chi}^{A}\right)^{k}{ }_{i}\left(T_{\xi}^{A}\right)^{l}{ }_{j}=-y_{a i j} \frac{C_{2}\left(R_{\chi}\right)+C_{2}\left(R_{\xi}\right)-C_{2}\left(R_{\phi}\right)}{2}$,

we arrive at

$$
\begin{aligned}
\delta y_{a i j}^{(1)}= & -y_{a i j} \frac{3}{2 \mathcal{N}} \frac{C_{2}\left(R_{\chi}\right)+C_{2}\left(R_{\xi}\right)-C_{2}\left(R_{\phi}\right)}{2 S_{2}\left(R_{\Psi}\right)} \\
& \times \int_{0}^{K} \mathrm{~d} x H_{y}\left(0, \frac{2}{3} x\right) .
\end{aligned}
$$

\section{Quartic vertex}

We evaluate the leading order gauge vertex contribution to the scalar self-coupling. Such contributions first appear at $1 / \mathcal{N}^{2}$, and in the Landau gauge the only contribution stems from the diagram of Fig. 7. All other types of diagrams, see Fig. 8, contain at least one three-point gauge insertion on an external scalar leg. Since the gauge propagator is transverse in the Landau gauge, any such coupling is proportional to the external momenta and vanishes when this is taken to 0 . Therefore these diagrams do not contribute to the vertex counterterm.

We proceed by computing the diagrams in the Abelian theory before considering the non-Abelian one as well as the semisimple gauge groups.

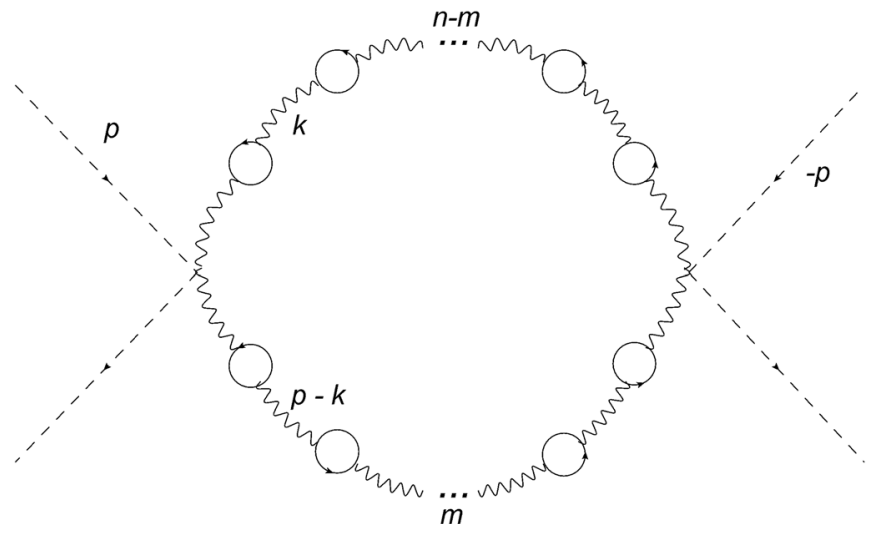

FIG. 7. $g^{4}$ vertex contribution.

\section{Abelian computation}

In order to evaluate the vertex contribution due to the diagrams in Fig. 7, we first denote by $i \Lambda^{(n, m)}$ such a diagram with $m$ bubbles on the one propagator and $n-m$ bubbles on the other. $\Lambda^{(n, m)}$ and $\Lambda^{(n, n-m)}$ are indistinguishable; therefore we include a factor of $\frac{1}{2}$ for each pair $(n, m)$ to avoid double counting. This also agrees with the diagrams where $n=2 m$, in which case the two bubble chains are indistinguishable and they receive a symmetry factor $\frac{1}{2}$ from the Feynman rules. In the limit of vanishing external momenta, all permutations of the scalar legs count the same. Moreover, in this limit, only the loop momenta pass through the bubble chains and the loop integrals are indifferent to which propagator the bubbles are placed on. The divergent part of the four-point function at vanishing external momenta is obtained from

$$
\begin{aligned}
\left.i \Lambda\right|_{1 / \epsilon} & =\left.\sum_{n=0}^{\infty} \sum_{m=0}^{n} i \Lambda^{(n, m)}\right|_{1 / \epsilon}+\text { permutation } \\
& =\left.2 \sum_{n=0}^{\infty}(n+1) i \Lambda^{(n, 0)}\right|_{1 / \epsilon} .
\end{aligned}
$$

It is thus clear that it is sufficient to evaluate only the diagrams with bubbles on one of the gauge lines.

To regulate the IR divergence of the relevant diagrams, we consider nonvanishing external momenta, as given in Fig. 7, from which we obtain
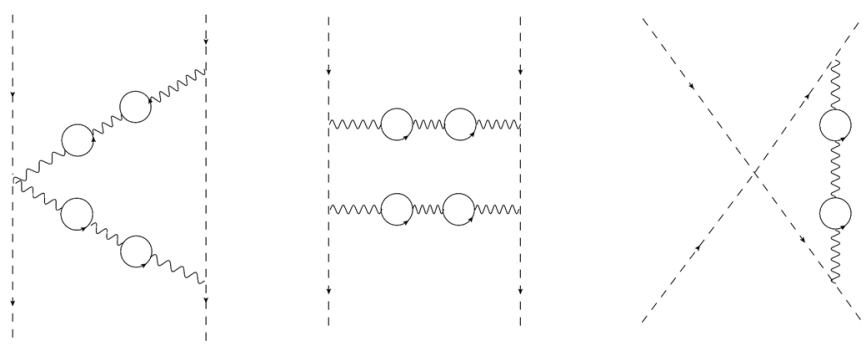

FIG. 8. $g^{4}$ with three-point vertex on the external scalar leg. 
$i \Lambda^{(n, 0)}=\frac{1}{2}\left(i 2 q_{\phi}^{2} \tilde{g}_{0}^{2}\right)^{2} \mu^{2 \epsilon} \int \frac{\mathrm{d}^{d} k}{(2 \pi)^{d}} D^{\mu \nu}(p-k) D_{\nu \mu}^{(n)}(k)$,

where the factor $\frac{1}{2}$ is the aforementioned symmetry factor. Evaluating the integral one obtains the result

$$
\begin{aligned}
i \Lambda^{(n, 0)}= & \frac{i \pi^{2}}{4 \mathcal{N}^{2}} \frac{q_{\phi}^{4}}{q_{\Psi}^{4}}\left(-2 K_{0}\right)^{n+2} \mu^{\epsilon} \\
& \times\left(-\frac{4 \pi \mu^{2}}{p^{2}}\right)^{(n+1) \epsilon / 2} \Gamma_{0}^{n}(\epsilon) \Gamma_{\lambda}(n, \epsilon),
\end{aligned}
$$

with

$$
\begin{aligned}
\Gamma_{\lambda}(n, \epsilon)= & \frac{(3-\epsilon)(4-\epsilon+n \epsilon)}{(n+1) \epsilon} \\
& \times \frac{\Gamma\left(1-\frac{n+1}{2} \epsilon\right) \Gamma\left(1-\frac{\epsilon}{2}\right) \Gamma\left(1+\frac{n+1}{2} \epsilon\right)}{\Gamma\left(2+\frac{n}{2} \epsilon\right) \Gamma\left(2-\frac{n+2}{2} \epsilon\right)} .
\end{aligned}
$$

At this point we may sum over all the different diagrams as indicated by Eq. (4.28) to obtain the pole structure of the vertex. By redefining the summation from $n \rightarrow n-2$, one finds

$\left.i \Lambda\right|_{1 / \epsilon}=\left.\frac{i 54 \pi^{2} \mu^{\epsilon}}{\mathcal{N}^{2}} \frac{q_{\phi}^{4}}{q_{\Psi}^{4}} \sum_{n=2}^{\infty}\left(-\frac{2 K_{0}}{3}\right)^{n} \frac{1}{\epsilon^{n-1}} H_{\lambda}(n, \epsilon)\right|_{1 / \epsilon}$,

where

$$
\begin{aligned}
H_{\lambda}(n, \epsilon)= & \left(-\frac{4 \pi \mu^{2}}{p^{2}}\right){ }^{(n-1) \epsilon / 2} \\
& \times\left[3 \epsilon \Gamma_{0}(\epsilon)\right]^{n-2} \frac{(n-1) \epsilon}{12} \Gamma_{\lambda}(n-2, \epsilon) .
\end{aligned}
$$

It is now possible to resum the pole structure of the vertex contribution using (A22). The resulting leading order gauge contribution to the quartic counterterm pole is

$$
\delta \lambda^{(1)}=\left.\Lambda\right|_{1 / \epsilon}=\frac{24 \pi^{2}}{\mathcal{N}^{2}} \frac{q_{\phi}^{4}}{q_{\Psi}^{4}} \mu^{\epsilon} K^{2} H_{\lambda}\left(1, \frac{2}{3} K\right),
$$

with

$$
H_{\lambda}(1, x)=\frac{\left(1-\frac{x}{3}\right) \Gamma(4-x)}{6 \Gamma^{3}\left(2-\frac{x}{2}\right) \Gamma\left(1+\frac{x}{2}\right)} .
$$

\section{Non-Abelian case}

In the non-Abelian case we have

$$
\begin{aligned}
i \Lambda^{(n, 0) a b}{ }_{c d}= & \frac{1}{2}\left(i \tilde{g}_{0}^{2}\right)^{2}\left\{T_{\phi}^{A}, T_{\phi}^{B}\right\}^{a}{ }_{c}\left\{T_{\phi}^{A}, T_{\phi}^{B}\right\}^{b}{ }_{d} \mu^{2 \epsilon} \\
& \times \int \frac{\mathrm{d}^{d} k}{(2 \pi)^{d}} D^{\mu \nu}(p-k) D_{\nu \mu}^{(n)}(k) .
\end{aligned}
$$

By comparing this expression with the Abelian diagram of (4.29) we can read off the non-Abelian result, paying

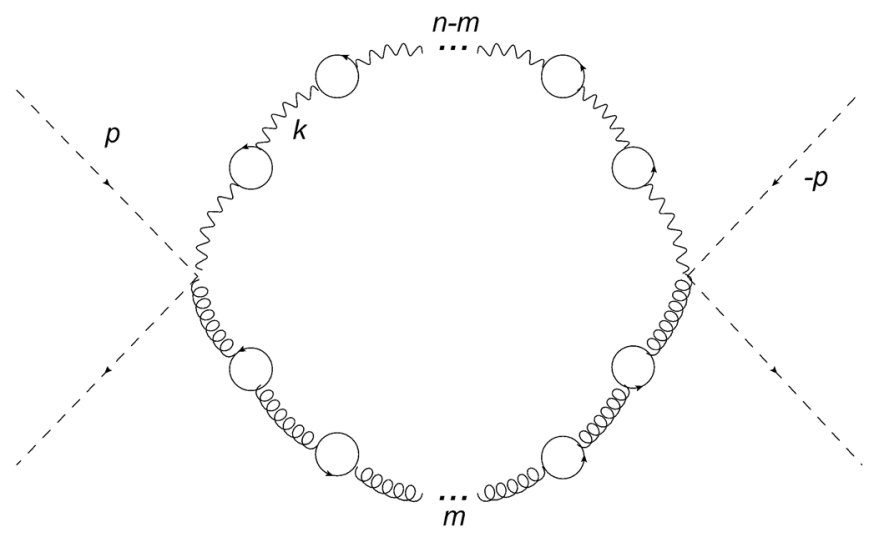

FIG. 9. The mixed gauge term contributing to the quartic vertex in a semisimple gauge theory.

attention to the fact that the color structure changes depending on the permutation of the external scalars. The contribution to the counterterm in the non-Abelian theory is thus given by

$$
\delta \lambda^{(1) a b}{ }_{c d}=\frac{24 \pi^{2} d^{2}\left(R_{\Psi}\right)}{\mathcal{N}^{2} S_{2}^{2}\left(R_{\Psi}\right)} A^{a b}{ }_{c d} K^{2} H_{\lambda}\left(1, \frac{2}{3} K\right),
$$

with

$$
\begin{aligned}
A^{a b}{ }_{c d}= & \frac{1}{8}\left(\left\{T_{\phi}^{A}, T_{\phi}^{B}\right\}_{c}^{a}\left\{T_{\phi}^{A}, T_{\phi}^{B}\right\}^{b}{ }_{d}\right. \\
& \left.+\left\{T_{\phi}^{A}, T_{\phi}^{B}\right\}^{a}{ }_{d}\left\{T_{\phi}^{A}, T_{\phi}^{B}\right\}_{c}^{b}\right) .
\end{aligned}
$$

\section{Semisimple gauge group}

The quartic coupling contains mixed gauge-coupling contributions already at $\mathrm{LO}$ in $1 / \mathcal{N}$. If we consider the case where both the scalar and the vectorlike fermions are charged under a semisimple gauge group, then the quartic coupling receives mixed contributions of the type sketched in Fig. 9. For every pair of simple gauge groups $\left(G_{\alpha}, G_{\beta}\right)$, all the diagrams contain at least one power of $K_{\alpha, 0}$ and $K_{\beta, 0}$, respectively. Starting from the simple diagram where all fermion bubbles are put on the $G_{\beta}$ gauge line, we have

$$
\begin{aligned}
i \Lambda^{(n, 0)}= & \left(i 2 \tilde{g}_{\alpha, 0} \tilde{g}_{\beta, 0}\right)^{2}\left(T_{\phi, \alpha}^{A} T_{\phi, \beta}^{B}\right)^{a}{ }_{c}\left(T_{\phi, \alpha}^{A} T_{\phi, \beta}^{B}\right)^{b} \mu^{2 \epsilon} \\
& \times \int \frac{\mathrm{d}^{d} k}{(2 \pi)^{d}} D_{\alpha}^{\mu \nu}(p-k) D_{\beta, \nu \mu}^{(n)}(k) .
\end{aligned}
$$

Recall here that the generators belong to different gauge groups and therefore they commute. Comparing this diagram to Eqs. (4.29) and (4.30) leads to

$$
\begin{aligned}
i \Lambda^{(n, 0)}= & i\left(T_{\phi, \alpha}^{A} T_{\phi, \beta}^{B}\right)_{c}^{a}\left(T_{\phi, \alpha}^{A} T_{\phi, \beta}^{B}\right)^{b} \frac{\pi^{2} \mu^{\epsilon}}{2 \mathcal{N}^{2}} \frac{d\left(R_{\Psi}^{\alpha}\right) d\left(R_{\Psi}^{\beta}\right)}{S_{2}\left(R_{\Psi}^{\alpha}\right) S_{2}\left(R_{\Psi}^{\beta}\right)} \\
& \times\left(-2 K_{\alpha, 0}\right)\left(-2 K_{\beta, 0}\right)^{n+1}\left(-\frac{4 \pi \mu^{2}}{p^{2}}\right)^{(n+1) \epsilon / 2} \\
& \times \Gamma_{0}^{n}(\epsilon) \Gamma_{\lambda}(n, \epsilon) .
\end{aligned}
$$


We can distribute the fermion bubbles in several ways on the two gauge lines. Each bubble gives a factor of $K_{\alpha, 0}$ or $K_{\beta, 0}$ depending on the gauge line, but the kinematic part of the diagram remains unchanged in the limit of vanishing external momentum. Because the pole structure does not depend on the external momentum, the pole structure of a general diagram can be related to the diagram with fermion bubbles on only one of the gauge lines. In particular,

$$
\left.i \Lambda^{(n, m)}\right|_{1 / \epsilon}=\left.i\left(\frac{K_{\alpha, 0}}{K_{\beta, 0}}\right)^{m} \Lambda^{(n, 0)}\right|_{1 / \epsilon} .
$$

Thus summing over all possible bubbles and taking into account the different permutations of the external scalars, we arrive at

$$
\left.i \Lambda_{c d}^{a b}\right|_{1 / \epsilon}=\left.i B_{\alpha, \beta}{ }^{a b}{ }_{c d} \frac{108 \pi^{2}}{\mathcal{N}^{2}} \frac{d\left(R_{\Psi}^{\alpha}\right) d\left(R_{\Psi}^{\beta}\right)}{S_{2}\left(R_{\Psi}^{\alpha}\right) S_{2}\left(R_{\Psi}^{\beta}\right)} \sum_{n=1}^{\infty} \sum_{m=0}^{n-1}\left(-\frac{2 K_{\alpha, 0}}{3}\right)^{1+m}\left(-\frac{2 K_{\beta, 0}}{3}\right)^{n-m} \frac{H_{\lambda}(n+1, \epsilon)}{n \epsilon^{n}}\right|_{1 / \epsilon},
$$

having defined the tensor

$$
B_{\alpha, \beta}{ }^{a b}{ }_{c d}=\frac{1}{2}\left[\left(T_{\phi, \alpha}^{A} T_{\phi, \beta}^{B}\right)_{c}^{a}\left(T_{\phi, \alpha}^{A} T_{\phi, \beta}^{B}\right)_{d}^{b}+\left(T_{\phi, \alpha}^{A} T_{\phi, \beta}^{B}\right)^{a}{ }_{d}\left(T_{\phi, \alpha}^{A} T_{\phi, \beta}^{B}\right)_{c}^{b}\right] .
$$

Employing Eq. (A29) and collecting the contributions from all the mixed terms, we find

$$
\delta \lambda^{(1) a b}{ }_{c d}=\sum_{\alpha} \frac{24 \pi^{2} d^{2}\left(R_{\Psi}^{\alpha}\right)}{\mathcal{N}^{2} S_{2}^{2}\left(R_{\Psi}^{\alpha}\right)} A_{1}{ }^{a b}{ }_{c d} K_{\alpha}^{2} H_{\lambda}\left(1, \frac{2}{3} K_{\alpha}\right)+\sum_{\alpha<\beta} B_{\alpha, \beta}{ }^{a b}{ }_{c d} \frac{48 \pi^{2}}{\mathcal{N}^{2}} \frac{d\left(R_{\Psi}^{\alpha}\right) d\left(R_{\Psi}^{\beta}\right)}{S_{2}\left(R_{\Psi}^{\alpha}\right) S_{2}\left(R_{\Psi}^{\beta}\right)} \frac{K_{\alpha} K_{\beta}}{K_{\alpha}-K_{\beta}} \int_{K_{\beta}}^{K_{\alpha}} \mathrm{d} x H_{\lambda}\left(1, \frac{2}{3} x\right),
$$

which naturally also contains the unmixed contributions.

\section{E. Complete set of large $\mathcal{N}$ beta functions}

Having evaluated all relevant diagrams we now compute all the beta functions using Eq. (2.10), starting with the Yukawa that reads

$$
\begin{aligned}
\left(\beta_{y}\right)_{a i j}= & \frac{1}{32 \pi^{2}}\left[\left(y_{b} y^{\dagger, b} y_{a}\right)_{i j}+\left(y_{a} y^{\dagger, b} y_{b}\right)_{i j}+2 \operatorname{Tr}\left[y_{a} y^{\dagger, b}\right] y_{b i j}\right] \\
& -y_{a i j} \sum_{\alpha} \frac{3 d\left(R_{\Psi}^{\alpha}\right)}{16 \mathcal{N}} \frac{C_{2}\left(R_{\chi}^{\alpha}\right)+C_{2}\left(R_{\xi}^{\alpha}\right)}{S_{2}\left(R_{\Psi}^{\alpha}\right)} K_{\alpha} H_{\psi}\left(0, \frac{2}{3} K_{\alpha}\right)-y_{a i j} \sum_{\alpha} \frac{3 d\left(R_{\Psi}^{\alpha}\right)}{4 \mathcal{N}} \frac{C_{2}\left(R_{\phi}^{\alpha}\right)}{S_{2}\left(R_{\Psi}^{\alpha}\right)} K_{\alpha} H_{\phi}\left(0, \frac{2}{3} K_{\alpha}\right) \\
& -y_{a i j} \sum_{\alpha} \frac{3 d\left(R_{\Psi}^{\alpha}\right)}{2 \mathcal{N}} \frac{C_{2}\left(R_{\chi}^{\alpha}\right)+C_{2}\left(R_{\xi}^{\alpha}\right)-C_{2}\left(R_{\phi}^{\alpha}\right)}{2 S_{2}\left(R_{\Psi}^{\alpha}\right)} K_{\alpha} H_{y}\left(0, \frac{2}{3} K_{\alpha}\right)+\mathcal{O}\left(\frac{1}{\mathcal{N}^{2}}\right) .
\end{aligned}
$$

This result includes the one-loop terms from the matter sector.

For the quartic scalar coupling, the beta function is

$$
\begin{aligned}
\beta_{\lambda}{ }^{a b}{ }_{c d}= & \frac{1}{16 \pi^{2}}\left(2 \lambda^{a e}{ }_{c f} \lambda^{b f}{ }_{d e}+2 \lambda^{a e}{ }_{d f} \lambda^{b f}{ }_{c e}+\lambda^{a b}{ }_{e f} \lambda^{e f}{ }_{c d}\right)+\frac{1}{4 \pi^{2}} \operatorname{Tr}\left[y_{d} y^{\dagger, e}\right] \lambda^{a b}{ }_{c e} \\
& -\frac{1}{4 \pi^{2}} \operatorname{Tr}\left[y^{a} y_{c}^{\dagger} y^{b} y_{d}^{\dagger}+y^{a} y_{d}^{\dagger} y^{b} y_{c}^{\dagger}\right]-\lambda^{a b}{ }_{c d} \sum_{\alpha} \frac{3 d\left(R_{\Psi}^{\alpha}\right)}{\mathcal{N}} \frac{C_{2}\left(R_{\phi}^{\alpha}\right)}{S_{2}\left(R_{\Psi}^{\alpha}\right)} K_{\alpha} H_{\phi}\left(0, \frac{2}{3} K_{\alpha}\right) \\
& +\frac{48 \pi^{2}}{\mathcal{N}^{2}} \sum_{\alpha<\beta} B_{\alpha, \beta}{ }^{a b}{ }_{c d} \frac{d\left(R_{\Psi}^{\alpha}\right) d\left(R_{\Psi}^{\beta}\right)}{S_{2}\left(R_{\Psi}^{\alpha}\right) S_{2}\left(R_{\Psi}^{\beta}\right)} \frac{K_{\alpha} K_{\beta}}{K_{\alpha}-K_{\beta}}\left[K_{\alpha} H_{\lambda}\left(1, \frac{2}{3} K_{\alpha}\right)-K_{\beta} H_{\lambda}\left(1, \frac{2}{3} K_{\beta}\right)\right] \\
& +\frac{24 \pi^{2}}{\mathcal{N}^{2}} \sum_{\alpha} A_{\alpha}{ }^{a b}{ }_{c d} \frac{d^{2}\left(R_{\Psi}^{\alpha}\right)}{S_{2}^{2}\left(R_{\Psi}^{\alpha}\right)}\left[K_{\alpha}^{2} H_{\lambda}\left(1, \frac{2}{3} K_{\alpha}\right)+K_{\alpha}^{3} \frac{\partial}{\partial K_{\alpha}} H_{\lambda}\left(1, \frac{2}{3} K_{\alpha}\right)\right]+\mathcal{O}\left(\frac{1}{\mathcal{N}^{3}}\right) .
\end{aligned}
$$

Finally the gauge beta function for the full model of Eq. (2.1) is

$$
\beta_{K_{\alpha}}=\frac{2 K_{\alpha}^{2}}{3}\left[1+\frac{d\left(R_{\Psi}^{\alpha}\right)}{\mathcal{N} S_{2}\left(R_{\Psi}^{\alpha}\right)}\left(\frac{1}{2} S_{2}\left(R_{\chi}^{\alpha}\right)+\frac{1}{2} S_{2}\left(R_{\xi}^{\alpha}\right)+\frac{1}{4} S_{2}\left(R_{\phi}^{\alpha}\right)\right)+\frac{d(G)}{\mathcal{N}} H_{1}^{(\alpha)}\left(K_{\alpha}\right)+\frac{1}{\mathcal{N}} \sum_{\beta \neq \alpha} d\left(G_{\beta}\right) F_{1}\left(K_{\beta}\right)\right]+\mathcal{O}\left(\frac{1}{\mathcal{N}^{2}}\right) .
$$


We note that the $1 / \mathcal{N}$ counting is consistent with the fact that $\lambda \sim y^{2} \sim g^{2} \sim 1 / \mathcal{N}$.

\section{F. A mnemonic for Yukawa and quartic beta functions}

Since the beta functions of many phenomenological models are known to LO, it is convenient to rewrite the above Yukawa and quartic beta function Eqs. (4.45) and (4.46) in a more compact form. With this prescription one can immediately obtain the bubble diagram contributions to known one-loop beta functions by simply using the following recipe. The Yukawa beta function at a large number of fermions can be written in the following compact form,

$$
\beta_{y}=c_{1} y^{3}+y \sum_{\alpha} c_{\alpha} K_{\alpha} I_{y}\left(K_{\alpha}\right),
$$

with

$I_{y}\left(K_{\alpha}\right)=H_{\phi}\left(0, \frac{2}{3} K_{\alpha}\right)\left(1+K_{\alpha} \frac{C_{2}\left(R_{\phi}^{\alpha}\right)}{6\left(C_{2}\left(R_{\chi}^{\alpha}\right)+C_{2}\left(R_{\xi}^{\alpha}\right)\right)}\right)$,

containing information about the resummed fermion bubbles and $c_{1}, c_{\alpha}$ are the standard one-loop coefficients for the Yukawa beta function. Thus, when $c_{1}, c_{\alpha}$ are known, the total Yukawa beta function with bubble diagram contributions is straightforward. Similarly, for the quartic coupling we write

$$
\begin{aligned}
\beta_{\lambda}= & c_{1} \lambda^{2}+\lambda \sum_{\alpha} c_{\alpha} K_{\alpha} I_{\lambda g^{2}}\left(K_{\alpha}\right)+\sum_{\alpha} c_{\alpha}^{\prime} K_{\alpha}^{2} I_{g^{4}}\left(K_{\alpha}\right) \\
& +\sum_{\alpha<\beta} c_{\alpha \beta} K_{\alpha} K_{\beta} I_{g_{1}^{2} g_{2}^{2}}\left(K_{\alpha}, K_{\beta}\right),
\end{aligned}
$$

with $c_{1}, c_{\alpha}, c_{\alpha}^{\prime}, c_{\alpha \beta}$ being the known one-loop coefficients ${ }^{6}$ for the quartic beta function, and the resummed fermion bubbles appear via

$$
\begin{aligned}
I_{\lambda g^{2}}\left(K_{\alpha}\right)= & H_{\phi}\left(0, \frac{2}{3} K_{\alpha}\right) \\
I_{g^{4}}\left(K_{\alpha}\right)= & H_{\lambda}\left(1, \frac{2}{3} K_{\alpha}\right)+K_{\alpha} \frac{d H_{\lambda}\left(1, \frac{2}{3} K_{\alpha}\right)}{d K_{\alpha}} \\
I_{g_{1}^{2} g_{2}^{2}}\left(K_{\alpha}, K_{\beta}\right)= & \frac{1}{K_{\alpha}-K_{\beta}}\left[K_{\alpha} H_{\lambda}\left(1, \frac{2}{3} K_{\alpha}\right)\right. \\
& \left.-K_{\beta} H_{\lambda}\left(1, \frac{2}{3} K_{\beta}\right)\right] .
\end{aligned}
$$

It is thus also straightforward to obtain the total quartic beta function including the bubble diagram contributions when $c_{1}, c_{\alpha}, c_{\alpha}^{\prime}, c_{\alpha \beta}$ are known.

\footnotetext{
${ }^{6}$ Clearly these are not the same numerical coefficients appearing in (4.48).
}

\section{G. Pole structure of the beta functions}

We now elucidate the pole structure of the resummed beta functions, which is a characteristic feature of the theories investigated here.

Since the pole structure of beta function in theories with a simple gauge group has been discussed already in the literature [29-32], we move immediately to consider the semisimple gauge-fermion theories. Here we observe that if the group structures contain an Abelian factor the corresponding beta function is such that it still features a singularity for $K=\frac{15}{2}$ regardless of the presence of other non-Abelian factors. This is so since the extra contribution assumes the form of $F_{1}$ rather than $H_{1}$ [see Eq. (4.46)]. Thus, it is not possible to shift the resulting UV fixed point value of the Abelian gauge coupling away from the Abelian pole. This is clearly manifest in the phase diagram structure of Fig. 3(b).

For the Yukawa beta function we first observe, using Eqs. (4.7), (4.16), and (4.24), that the following relations hold:

$$
\begin{aligned}
& H_{\psi}(0, x)=x H_{0}(x), \quad H_{\phi}(0, x)=H_{0}(x), \\
& H_{y}(0, x)=H_{\lambda}(1, x)=\left(1-\frac{x}{4}\right) H_{0}(x),
\end{aligned}
$$

where

$$
H_{0}(x)=\frac{\left(1-\frac{x}{3}\right) \Gamma(4-x)}{3 \Gamma^{2}\left(2-\frac{x}{2}\right) \Gamma\left(3-\frac{x}{2}\right) \Gamma\left(1+\frac{x}{2}\right)} .
$$

This means that they all inherit a pole at $x=5$ yielding

$$
\begin{aligned}
H_{\psi}\left(0, \frac{2}{3} K_{\alpha}\right) & \sim \frac{1}{\frac{15}{2}-K_{\alpha}}, \quad H_{\phi}\left(0, \frac{2}{3} K_{\alpha}\right) \sim \frac{1}{\frac{15}{2}-K_{\alpha}}, \\
H_{y}\left(0, \frac{2}{3} K_{\alpha}\right) & \sim \frac{1}{K_{\alpha}-\frac{15}{2}} .
\end{aligned}
$$

Thus the Yukawa coupling RG function Eq. (4.45) near the pole assumes the following form:

$$
\beta_{y}=c_{1} y^{3}+y K_{\alpha}\left(\frac{1}{K_{\alpha}-\frac{15}{2}}\right)\left(c_{2}+c_{3} K_{\alpha}\right),
$$

where $c_{1}, c_{2}, c_{3}$ are positive constants stemming from the group structure of the theory. It is clear that the three summation functions altogether provide large negative contributions when approaching the pole (i.e., $K_{\alpha}=\frac{15}{2}$ ) from the left. The pole in the Yukawa beta function appears at the original Abelian gauge coupling location [see Eq. (3.12)]. This implies that if the gauge group features an Abelian factor, from Eq. (4.55), we deduce that the Yukawa gauge coupling vanishes in the UV (free rather than safe). The situation changes dramatically when only non-Abelian gauge groups are involved. This is so since the non-Abelian gauge beta function reaches an UV fixed point at $K_{\alpha}=3$, which is clearly 
away from the Abelian pole, allowing for nontrivial UV 0's of the Yukawa beta function.

Similarly to the Yukawa beta function the RG equation for the quartic coupling, due to the $K_{\alpha} H_{\phi}\left(0, \frac{2}{3} K_{\alpha}\right)$ term in Eq. (4.46), receives a large negative contribution at the Abelian pole. This is made explicit by the relations

$$
\begin{aligned}
H_{\lambda}\left(1, \frac{2}{3} K_{\alpha}\right) & =H_{y}\left(0, \frac{2}{3} K_{\alpha}\right) \sim \frac{1}{K_{\alpha}-\frac{15}{2}}, \\
\frac{\partial}{\partial K_{\alpha}} H_{\lambda}\left(1, \frac{2}{3} K_{\alpha}\right) & \sim-\frac{1}{\left(K_{\alpha}-\frac{15}{2}\right)^{2}} .
\end{aligned}
$$

Thus, the quartic coupling RG function Eq. (4.46) near the first singularity assumes the form

$\beta_{\lambda}=c_{1} \lambda^{2}+c_{2} \lambda K_{\alpha}\left(\frac{1}{K_{\alpha}-\frac{15}{2}}\right)+c_{3} K_{\alpha}^{2}\left(\frac{1}{K_{\alpha}-\frac{15}{2}}-\frac{1}{\left(K_{\alpha}-\frac{15}{2}\right)^{2}}\right)$,

where $c_{1}, c_{2}, c_{3}$ denote positive constants (distinct from the Yukawa case). From Eq. (4.57) we learn that the quartic beta function has an even more singular structure located at the Abelian pole. If the theory contains an Abelian gauge group one observes that the quartic coupling develops an explosive behavior $\left(\lambda \propto \exp \left(N_{f}\right)\right)$ at the Abelian fixed point and the fixed point analysis cannot be trusted.

The situation for the non-Abelian case resemble the Yukawa case. Here the non-Abelian UV fixed point is achieved at $K_{\alpha}=3$ which is below and sufficiently away from the pole in the quartic coupling, allowing for (depending on the theory) the existence of UV fixed points in all couplings.

\section{CONCLUSION}

We investigated gauge-Yukawa theories at a large number of gauged fermion fields. We began our analysis by reviewing the state of the art of the gauge-fermion theories. We considered also semisimple groups and by discussing their RG phase diagram we discovered a complete asymptotically safe fixed point which turns out to be repulsive in all gauge couplings.

Subsequently we enriched the original gauge-fermion theories by introducing two Weyl gauged fermions transforming according to arbitrary representations of the gauge group and further added a complex gauged scalar. The latter is responsible for the presence of Yukawa and quartic scalar self-coupling interactions. On par with the gauge sector, we determined the leading $1 / N_{f}$ Yukawa and quartic beta functions. We then discussed the pole structure of the system of RG equations. This has an immediate impact on the existence, location and stability of related fixed points. In particular, one observes that when an Abelian gauge coupling is present in the theory, the Yukawa beta function is driven to be free while the quartic coupling becomes uncontrollable, de facto requiring a fully nonperturbative analysis near this point. The situation changes dramatically when only non-Abelian gauge couplings are present. Because the latter achieve a fixed point at a much lower value of the Abelian one (still appearing as the only pole in the Yukawa and quartic beta function) Yukawa and quartic couplings (depending on the theory) can still admit UV interacting fixed points. These results cannot be extended to the supersymmetric case [23] for a number of reasons. The first reason is that the resummation procedure would have to respect supersymmetry and, in addition, it has already been proven in [23] that it is impossible to have an UV fixed point for any $N_{f}$ in super-QCD.

Our work elucidates, corrects, consolidates, and extends results obtained earlier in the literature [13,14,27-32,35]. It also provides the stepping stone and the needed instruments for future theoretical and phenomenological extensions and analyses.

\section{ACKNOWLEDGMENTS}

The work of N. A. D., A.E. T., F. S., and Z.-W. W. is partially supported by the Danish National Research Foundation under the Grant No. DNRF:90, the Croatian Science Foundation under Grant No. 4418 and the Natural Sciences and Engineering Research Council of Canada (NSERC). O. A. also acknowledges the partial support by the H2020 CSA Twinning Grant No. 692194, RBI-TWINNING. Z.-W. W. thanks Robert Mann, Tom Steele, Cacciapaglia Giacomo and Emiliano Molinaro for helpful suggestions.

\section{APPENDIX: RESUMMATION FORMULAS}

Here we present proofs for the four resummation formulas used for the large $\mathcal{N}$ computations. Regardless of the quantity in question only the pole structure at $\epsilon \rightarrow 0$ is relevant. We use the notation " $\underset{\epsilon \rightarrow 0}{\sim}$ " to mean equal divergent parts. Note that all of these resummations are only valid to $\mathrm{LO}$ in $1 / \mathcal{N}$.

All the resummation formulas rely on a function $H(n, \epsilon)$ being regular both for $\alpha=n \epsilon \rightarrow 0$ with $\epsilon$ constant and for $\epsilon \rightarrow 0$ with $\alpha$ constant. In general, the functions occurring in this paper can easily be checked to satisfy this condition, except, possibly, for the term $\left[3 \epsilon \Gamma_{0}(\epsilon)\right]^{n}$ with fixed $\alpha$ and $\epsilon \rightarrow 0$. To see that this term is well behaved, it is sufficient to note that the base satisfies

$$
\left(\frac{6 \Gamma^{2}\left(2-\frac{\epsilon}{2}\right) \Gamma\left(1+\frac{\epsilon}{2}\right)}{\Gamma(4-\epsilon)}\right)^{\alpha / \epsilon-1}=(1+\epsilon f(\epsilon))^{\alpha / \epsilon},
$$

for some regular function $f$ that is regular in 0 . To prove that this expression has no pole, observe that there must exist constants $c, \delta>0$ such that

$$
|f(\epsilon)| \leq c, \quad \forall|\epsilon|<\delta .
$$

It must then hold that 


$$
\lim _{\epsilon \rightarrow 0}|1+\epsilon f(\epsilon)|^{\alpha / \epsilon} \leq \lim _{\epsilon \rightarrow 0_{+}}(1+c \epsilon)^{\alpha / \epsilon}=\lim _{x \rightarrow \infty}\left(1+\frac{c}{x}\right)^{x \alpha}=e^{c \alpha}
$$

having substituted $x=\frac{1}{\epsilon}$. It is thus found that $\left[3 \epsilon \Gamma_{0}(\epsilon)\right]^{n}$ is without a pole in $\epsilon \rightarrow 0$ and fixed $n \epsilon$.

\section{First resummation}

$$
\sum_{n=1}^{\infty}\left(-\frac{2 K_{0}}{3}\right)^{n} \frac{H(n+1, \epsilon)}{(n+1) \epsilon^{n}} \underset{1 / \epsilon}{\sim}-\frac{2}{3 \epsilon} \int_{0}^{K} \mathrm{~d} x\left(1-\frac{x}{K}\right) H\left(0, \frac{2}{3} x\right)
$$

The proof, originally due to [27], is presented here to keep the work self-contained. We define

$$
R(\epsilon)=\sum_{n=1}^{\infty}\left(-\frac{2 K_{0}}{3}\right)^{n} \frac{H(n+1, \epsilon)}{(n+1) \epsilon^{n}} .
$$

The resummation proceeds assuming that $H(n, \epsilon)$ can be written as a power series,

$$
H(n, \epsilon)=\sum_{j=0}^{\infty} H^{(j)}(\epsilon)(n \epsilon)^{j},
$$

where every $H^{(j)}(\epsilon)$ is regular in $\epsilon$. Such an expansion only exists if $H\left(\frac{\alpha}{\epsilon}, \epsilon\right)$ is regular in both $\alpha$ and $\epsilon$. Furthermore, the bare coupling is renormalized with $K_{0}=Z_{K}^{-1} K$. As this is a LO in $1 / \mathcal{N}$ computation, one may then expand

$$
\begin{aligned}
Z_{K}^{-n} & =\left[1-\frac{2 K}{3 \epsilon}+O\left(\frac{1}{\mathcal{N}}\right)\right]^{-n} \\
& =\sum_{k=0}^{\infty}\left(\begin{array}{c}
n+k-1 \\
k
\end{array}\right)\left(\frac{2 K}{3 \epsilon}\right)^{k}+O\left(\frac{1}{\mathcal{N}}\right) .
\end{aligned}
$$

Inserting all this back into Eq. (A5), we find

$$
\begin{aligned}
R(\epsilon)= & \sum_{n=1}^{\infty} \sum_{k=0}^{\infty} \sum_{j=0}^{\infty}\left(-\frac{2 K}{3}\right)^{n+k}\left(\begin{array}{c}
n+k-1 \\
k
\end{array}\right) \\
& \times \frac{(-1)^{k}(n+1)^{j-1}}{\epsilon^{n+k-j}} H^{(j)}(\epsilon),
\end{aligned}
$$

where $H^{(j)}(\epsilon)$ are regular functions of $\epsilon$. Defining $m=n+k$, the sums are redefined in terms of $m$ and $k$. The only poles in $\epsilon$ occur for $j<m$, so the divergent part of $R$ is given by

$$
\begin{aligned}
R(\epsilon) \underset{\epsilon \rightarrow 0}{\sim} & \sum_{m=1}^{\infty}\left(-\frac{2 K}{3}\right)^{m} \sum_{j=0}^{m-1} \frac{H_{j}(\epsilon)}{\epsilon^{m-j}} \\
& \times \sum_{k=0}^{m-1}\left(\begin{array}{c}
m-1 \\
k
\end{array}\right)(-1)^{k}(m-k+1)^{j-1} .
\end{aligned}
$$

The sum greatly simplifies as the identity

$$
\sum_{k=0}^{m}\left(\begin{array}{c}
m \\
k
\end{array}\right)(-1)^{k}(x-k)^{j}=0
$$

valid for all integer $0 \leq j<m$ and real numbers $x$, implies that the $k$ sum vanishes for all $j \neq 0$. Meanwhile the $j=0$ term evaluates to

$$
\sum_{k=0}^{m-1}\left(\begin{array}{c}
m-1 \\
k
\end{array}\right) \frac{(-1)^{k}}{m-k+1}=\frac{(-1)^{m-1}}{m(m+1)} .
$$

Performing the $j$ and $k$ sums then yields

$$
R(\epsilon) \underset{\epsilon \rightarrow 0}{\sim}-\sum_{m=1}^{\infty}\left(\frac{2 K}{3}\right)^{m} \frac{H^{(0)}(\epsilon)}{\epsilon^{m}} \frac{1}{m(m+1)} .
$$

Expanding now $H^{(0)}(\epsilon)=\sum_{\ell=0}^{\infty} H_{\ell}^{(0)} \epsilon^{\ell}$ and selecting the simple pole one finds

$$
R(\epsilon) \underset{1 / \epsilon}{\sim}-\frac{1}{\epsilon} \sum_{\ell=0}^{\infty}\left(\frac{2 K}{3}\right)^{\ell+1} \frac{H_{\ell}^{(0)}}{(\ell+1)(\ell+2)}
$$

Finally, before resumming the power series in $H_{\ell}^{(0)}$, the fraction is decomposed so that

$$
\begin{aligned}
R(\epsilon) & \underset{1 / \epsilon}{\sim}-\frac{1}{\epsilon}\left[\sum_{\ell=0}^{\infty}\left(\frac{2 K}{3}\right)^{\ell+1} \frac{H_{\ell}^{(0)}}{(\ell+1)}-\frac{3}{2 K} \sum_{\ell=0}^{\infty}\left(\frac{2 K}{3}\right)^{\ell+2} \frac{H_{\ell}^{(0)}}{(\ell+2)}\right] \\
\widetilde{1 / \epsilon} & -\frac{2}{3 \epsilon}\left[\int_{0}^{K} \mathrm{~d} x H\left(0, \frac{2}{3} x\right)-\frac{1}{K} \int_{0}^{K} \mathrm{~d} x x H\left(0, \frac{2}{3} x\right)\right],
\end{aligned}
$$

taking a $K$ derivative and resumming the power series before integrating again. This concludes the proof. 
2. Second resummation

$\sum_{n=1}^{\infty}\left(-\frac{2 K_{0}}{3}\right)^{n} \frac{1}{n \epsilon^{n}} H(n, \epsilon) \underset{1 / \epsilon}{\sim}-\frac{2}{3 \epsilon} \int_{0}^{K} \mathrm{~d} x H\left(0, \frac{2}{3} x\right)$

(A15)

To prove this formula [35], let

$$
S(\epsilon)=\sum_{n=1}^{\infty}\left(-\frac{2 K_{0}}{3}\right)^{n} \frac{1}{n \epsilon^{n}} H(n, \epsilon) .
$$

Assuming again that $H(n, \epsilon)$ is sufficiently regular, it may be expanded according to Eq. (A6). At the same time, the bare coupling is renormalized by $K_{0}=Z_{K}^{-1} K$. Then using the expansion Eq. (A7) one finds that

$$
\begin{aligned}
S(\epsilon)= & \sum_{n=1}^{\infty} \sum_{k=0}^{\infty} \sum_{j=0}^{\infty}\left(-\frac{2 K}{3}\right)^{n+k}\left(\begin{array}{c}
n+k-1 \\
k
\end{array}\right) \\
& \times \frac{(-1)^{k} n^{j-1}}{\epsilon^{n+k-j}} H^{(j)}(\epsilon) .
\end{aligned}
$$

Note that as $H^{j}(\epsilon)$ is regular in 0 , only the terms in the sum with $j \leq n+k-1$ contribute to the pole structure. The sums are redefined with $m=n+k$ and so

$$
\begin{aligned}
S(\epsilon) \underset{\epsilon \rightarrow 0}{\sim} & \sum_{m=1}^{\infty}\left(-\frac{2 K}{3}\right)^{m} \sum_{j=0}^{m-1} \frac{H^{(j)}(\epsilon)}{\epsilon^{m-j}} \\
& \times \sum_{k=0}^{m-1}\left(\begin{array}{c}
m-1 \\
k
\end{array}\right)(-1)^{k}(m-k)^{j-1} .
\end{aligned}
$$

According to the identity (A10), the $k$ sum vanishes for all $j \neq 0$. The sum is thus evaluated by keeping just the $j=0$ term, $^{7}$

$$
S\left(\epsilon \underset{\epsilon \rightarrow 0}{\sim}-\sum_{m=1}^{\infty}\left(\frac{2 K}{3}\right)^{m} \frac{1}{m} \frac{H^{(0)}(\epsilon)}{\epsilon^{m}} .\right.
$$

Expanding $H^{(0)}(\epsilon)$ as power series, the sum gives

$$
\begin{aligned}
& { }^{7} \text { For } j=0 \text { one has to use the summation, } \\
& \begin{aligned}
\sum_{k=0}^{n}\left(\begin{array}{l}
n \\
k
\end{array}\right) \frac{(-1)^{k}}{n-k+1} & =(-1)^{n} \sum_{k=0}^{n}\left(\begin{array}{l}
n \\
k
\end{array}\right) \frac{(-1)^{k}}{k+1} \\
& =\frac{(-1)^{n}}{n+1} \sum_{k=0}^{n}\left(\begin{array}{l}
n+1 \\
k+1
\end{array}\right)(-1)^{k}=\frac{(-1)^{n}}{n+1} .
\end{aligned}
\end{aligned}
$$

$$
\begin{aligned}
S(\epsilon) \underset{\epsilon \rightarrow 0}{\sim}-\sum_{m=1}^{\infty} \sum_{\ell=0}^{\infty}\left(\frac{2 K}{3}\right)^{m} \frac{1}{m} \frac{H_{\ell}^{(0)}}{\epsilon^{m-\ell}}, \text { where } \\
H^{(0)}(\epsilon)=\sum_{\ell=0}^{\infty} H_{\ell}^{(0)} \epsilon^{\ell} .
\end{aligned}
$$

As only the simple pole in epsilon is of interest to us, the resummation can now be concluded,

$$
\begin{aligned}
& S(\epsilon) \underset{1 / \epsilon}{\sim}-\frac{1}{\epsilon} \sum_{\ell=0}^{\infty}\left(\frac{2 K}{3}\right)^{\ell+1} \frac{1}{\ell+1} H_{\ell}^{(0)} \\
& \underset{1 / \epsilon}{\sim}-\frac{2}{3 \epsilon} \int_{0}^{K} \mathrm{~d} x \sum_{\ell=0}^{\infty}\left(\frac{2 x}{3}\right)^{\ell} H_{\ell}^{(0)} .
\end{aligned}
$$

The second to last step is done by taking a $K$ derivative before reintegrating so that the power series of $H^{(0)}(x)=$ $H(0, x)$ can finally be resummed proving (A15).

\section{Third resummation}

$\sum_{n=2}^{\infty}\left(-\frac{2 K_{0}}{3}\right)^{n} \frac{1}{\epsilon^{n-1}} H(n, \epsilon) \underset{1 / \epsilon}{\sim} \frac{1}{\epsilon}\left(\frac{2 K}{3}\right)^{2} H\left(1, \frac{2}{3} K\right)$

To prove that this is the case, let

$$
T(\epsilon)=\sum_{n=2}^{\infty}\left(-\frac{2 K_{0}}{3}\right)^{n} \frac{1}{\epsilon^{n-1}} H(n, \epsilon) .
$$

Here too, the function $H(n, \epsilon)$ is expanded as a power series in $n \epsilon$ as given in Eq. (A6) such that all $H^{(j)}(\epsilon)$ are regular in $\epsilon=0$. Simultaneously the bare couplings are renormalized $K_{0}=Z_{K}^{-1} K_{0}$ using Eq. (A7), and we write

$$
\begin{aligned}
T(\epsilon)= & \sum_{n=2}^{\infty} \sum_{j=0}^{\infty} \sum_{k=0}^{\infty}\left(-\frac{2 K}{3}\right)^{n+k}\left(\begin{array}{c}
n+k-1 \\
k
\end{array}\right)(-1)^{k} H^{(j)}(\epsilon) \\
& \times \frac{n^{j}}{\epsilon^{n+k-j-1}} .
\end{aligned}
$$

The sums can be rewritten in terms of $m=n+k$ so that

$$
\begin{aligned}
T(\epsilon)= & \sum_{m=2}^{\infty}\left(-\frac{2 K}{3}\right)^{m} \sum_{j=0}^{\infty} \frac{H^{(j)}(\epsilon)}{\epsilon^{m-j-1}} \\
& \times\left[\sum_{k=0}^{m-1}\left(\begin{array}{c}
m-1 \\
k
\end{array}\right)(-1)^{k}(m-k)^{j}-(-1)^{m-1}\right] .
\end{aligned}
$$


Here the last term is a compensation for the fact that the $k$ sum is taken to go all the way to $m-1$ rather than just to $m-2$. Poles in $\epsilon$ only appear for $j \leq m-2$ in which case the identity (A10) causes the first term in the sum to vanish. Thus, for the pole structure it holds that

$T(\epsilon) \underset{\epsilon \rightarrow 0}{\sim} \sum_{m=2}^{\infty}\left(\frac{2 K}{3}\right)^{m} \sum_{j=0}^{\infty} \frac{H^{(j)}(\epsilon)}{\epsilon^{m-j-1}}=\sum_{m=2}^{\infty}\left(\frac{2 K}{3}\right)^{m} \frac{H(1, \epsilon)}{\epsilon^{m-1}}$,

where the last equality is due to a resummation of the $H$ function. Reexpanding now $H(1, \epsilon)$ yields

$$
\begin{aligned}
T(\epsilon) \underset{\epsilon \rightarrow 0}{\sim}\left(\frac{2 K}{3}\right)^{2} \sum_{m=0}^{\infty} \sum_{\ell=0}^{\infty}\left(\frac{2 K}{3}\right)^{m} \frac{H_{\ell}}{\epsilon^{m+1-\ell}}, \text { where } \\
H(1, \epsilon)=\sum_{\ell=0}^{\infty} H_{\ell} \epsilon^{\ell} .
\end{aligned}
$$

At this point one can immediately determine the simple $\epsilon$ pole, which is evaluated to

$$
T(\epsilon) \underset{1 / \epsilon}{\sim} \frac{1}{\epsilon}\left(\frac{2 K}{3}\right)^{2} \sum_{m=0}^{\infty}\left(\frac{2 K}{3}\right)^{m} H_{m}=\frac{1}{\epsilon}\left(\frac{2 K}{3}\right)^{2} H\left(1, \frac{2}{3} K\right) .
$$

\section{Fourth resummation}

$$
\sum_{n=1}^{\infty} \sum_{m=0}^{n-1}\left(-\frac{2 K_{\alpha, 0}}{3}\right)^{1+m}\left(-\frac{2 K_{\beta, 0}}{3}\right)^{n-m} \frac{H(n, \epsilon)}{n \epsilon^{n}} \underset{1 / \epsilon}{\sim} \frac{4}{9 \epsilon} \frac{K_{\alpha} K_{\beta}}{K_{\alpha}-K_{\beta}} \int_{K_{\beta}}^{K_{\alpha}} \mathrm{d} x H\left(0, \frac{2}{3} x\right)
$$

To prove this, denote by

$$
U(\epsilon)=\sum_{n=1}^{\infty} \sum_{m=0}^{n-1}\left(-\frac{2 K_{\alpha, 0}}{3}\right)^{1+m}\left(-\frac{2 K_{\beta, 0}}{3}\right)^{n-m} \frac{H(n, \epsilon)}{n \epsilon^{n}}
$$

First the sum over $m$ is performed by noting that

$$
\sum_{m=0}^{n-1}\left(-\frac{2 K_{\alpha, 0}}{3}\right)^{1+m}\left(-\frac{2 K_{\beta, 0}}{3}\right)^{n-m}=\frac{2}{3}\left[\frac{1}{K_{\alpha, 0}}-\frac{1}{K_{\beta, 0}}\right]^{-1}\left[\left(-\frac{2 K_{\alpha, 0}}{3}\right)^{n}-\left(-\frac{2 K_{\beta, 0}}{3}\right)^{n}\right] .
$$

Now going from the bare to the renormalized couplings using $K_{\alpha, 0}=Z_{K_{\alpha}}^{-1} K_{\alpha}=K_{\alpha}\left(1-\frac{2 K_{\alpha}}{3 \epsilon}\right)^{-1}$ at LO in $1 / \mathcal{N}$, it is beneficial to first consider the first term,

$$
\left[\frac{1}{K_{\alpha, 0}}-\frac{1}{K_{\beta, 0}}\right]^{-1}=-\frac{K_{\alpha} K_{\beta}}{K_{\alpha}-K_{\beta}}
$$

This term is thus finite and does not contribute to the pole structure. The sum can be written as

$$
U(\epsilon)=-\frac{2}{3} \frac{K_{\alpha} K_{\beta}}{K_{\alpha}-K_{\beta}} \sum_{n=1}^{\infty}\left[\left(-\frac{2 K_{\alpha, 0}}{3}\right)^{n}-\left(-\frac{2 K_{\beta, 0}}{3}\right)^{n}\right] \frac{H(n, \epsilon)}{n \epsilon^{n}} .
$$

At this stage the resummation (A15) can be applied directly to obtain

$$
U(\epsilon) \underset{1 / \epsilon}{\sim} \frac{4}{9 \epsilon} \frac{K_{\alpha} K_{\beta}}{K_{\alpha}-K_{\beta}}\left[\int_{0}^{K_{\alpha}} \mathrm{d} x H\left(0, \frac{2}{3} x\right)-\int_{0}^{K_{\beta}} \mathrm{d} x H\left(0, \frac{2}{3} x\right)\right] .
$$


[1] K. G. Wilson, Phys. Rev. B 4, 3174 (1971).

[2] K. G. Wilson, Phys. Rev. B 4, 3184 (1971).

[3] D. J. Gross and F. Wilczek, Phys. Rev. D 8, 3633 (1973).

[4] H. D. Politzer, Phys. Rev. Lett. 30, 1346 (1973).

[5] D. F. Litim and F. Sannino, J. High Energy Phys. 12 (2014) 178.

[6] F. Sannino and V. Skrinjar, arXiv:1802.10372.

[7] D. F. Litim, M. Mojaza, and F. Sannino, J. High Energy Phys. 01 (2016) 081.

[8] F. Sannino, Proceedings, High-Precision Alpha Strong Measurements from LHC to FCC-ee: Geneva, Switzerland, 2015 (CERN, 2015), pp. 11-19.

[9] F. Sannino and I. M. Shoemaker, Phys. Rev. D 92, 043518 (2015).

[10] S. Abel and F. Sannino, Phys. Rev. D 96, 056028 (2017).

[11] S. Abel and F. Sannino, Phys. Rev. D 96, 055021 (2017).

[12] G. M. Pelaggi, F. Sannino, A. Strumia, and E. Vigiani, Front. Phys. 5, 49 (2017).

[13] R. Mann, J. Meffe, F. Sannino, T. Steele, Z.-W. Wang, and C. Zhang, Phys. Rev. Lett. 119, 261802 (2017).

[14] G. M. Pelaggi, A. D. Plascencia, A. Salvio, F. Sannino, J. Smirnov, and A. Strumia, Phys. Rev. D 97, 095013 (2018).

[15] A. D. Bond, G. Hiller, K. Kowalska, and D. F. Litim, J. High Energy Phys. 08 (2017) 004.

[16] A. Eichhorn, A. Held, and P. V. Griend, arXiv:1802.08589.

[17] A. Eichhorn, A. Held, and C. Wetterich, Phys. Lett. B 782, 198 (2018).

[18] M. Reichert, A. Eichhorn, H. Gies, J. M. Pawlowski, T. Plehn, and M. M. Scherer, Phys. Rev. D 97, 075008 (2018).

[19] A. Eichhorn, S. Lippoldt, and V. Skrinjar, Phys. Rev. D 97, 026002 (2018).

[20] A. Eichhorn and F. Versteegen, J. High Energy Phys. 01 (2018) 030.
[21] K. A. Intriligator and B. Wecht, Nucl. Phys. B667, 183 (2003).

[22] D. M. Hofman and J. Maldacena, J. High Energy Phys. 05 (2008) 012.

[23] K. Intriligator and F. Sannino, J. High Energy Phys. 11 (2015) 023.

[24] S. P. Martin and J. D. Wells, Phys. Rev. D 64, 036010 (2001).

[25] B. Bajc and F. Sannino, J. High Energy Phys. 12 (2016) 141.

[26] B. Bajc, N. A. Dondi, and F. Sannino, J. High Energy Phys. 03 (2018) 005.

[27] A. Palanques-Mestre and P. Pascual, Commun. Math. Phys. 95, 277 (1984).

[28] J. A. Gracey, Phys. Lett. B 373, 178 (1996).

[29] B. Holdom, Phys. Lett. B 694, 74 (2010).

[30] C. Pica and F. Sannino, Phys. Rev. D 83, 035013 (2011).

[31] R. Shrock, Phys. Rev. D 89, 045019 (2014).

[32] O. Antipin and F. Sannino, arXiv:1709.02354.

[33] F. Sannino, in Proceedings, 49th Cracow School of Theoretical Physics, Zakopane, Poland, 2009 [Acta Phys. Pol. B 40, 3533 (2009)].

[34] C. Pica, Proc. Sci., LATTICE2016 (2016) 015.

[35] K. Kowalska and E. M. Sessolo, J. High Energy Phys. 04 (2018) 027.

[36] P. M. Ferreira, I. Jack, and D. R. T. Jones, Phys. Lett. B 399, 258 (1997).

[37] P. M. Ferreira, I. Jack, D. R. T. Jones, and C. G. North, Nucl. Phys. B504, 108 (1997).

[38] L. Mihaila, in Proceedings, 11th International Symposium on Radiative Corrections (RADCOR 2013), Durham, United Kingdom, 2013 [Proc. Sci. RADCOR2013 (2013) 060].

[39] J. K. Esbensen, T. A. Ryttov, and F. Sannino, Phys. Rev. D 93, 045009 (2016). 